SUPPORTING INFORMATION

\title{
Synthesis of Highly Functionalized Macrocycles by the Peripheral Functionalization of Macrocyclic Diimines
}

\author{
Miguel A. Sierra,* Daniel Pellico, Mar Gómez-Gallego,* María José Mancheño, \\ and Rosario Torres ${ }^{\phi}$ \\ Departamento de Química Orgánica and Laboratorio de Difracción de Rayos X, Facultad de Química, \\ Universidad Complutense, 28040-Madrid, Spain
}

\section{Table of Contents}

1. General procedure for the synthesis of diimines 4-7

2. X-Ray diffraction analysis data

3. NMR spectra of compounds $4-22$ 


\section{General procedure for the synthesis of diimines (4 to 7):}

The corresponding dialdehyde was dissolved in $\mathrm{MeOH}$ or $\mathrm{EtOH}$, and the solution was purged with argon. A solution of the diamine in $\mathrm{MeOH}$ was added dropwise and the mixture was refluxed under argon for 3 to 24 hours, (the solution became yellow). The mixture was cooled to room temperature, filtered and the solvent was removed at reduced pressure.

Synthesis of diimine $4:^{2}$ A mixture of dialdehyde $1(1.0 \mathrm{~g}, 3.7 \mathrm{mmol})$ in $200 \mathrm{~mL}$ $\mathrm{MeOH}$ and 1,3-diaminopropane $(0.27 \mathrm{~g}, 3.7 \mathrm{mmol})$ in $50 \mathrm{~mL} \mathrm{MeOH}$, was stirred and refluxed for 3 hours. The solvent was removed at reduced pressure affording $1.09 \mathrm{~g}$ (94\%) of a pale yellow solid. mp $148-152{ }^{\circ} \mathrm{C}\left(\mathrm{EtOH}-\mathrm{CHCl}_{3}\right)\left(\mathrm{lit}^{2}{ }^{149-151}{ }^{\circ} \mathrm{C}\right) .{ }^{1} \mathrm{H}-$ NMR (200 MHz, $\left.\mathrm{CDCl}_{3}\right) \delta=8.72(\mathrm{~s}, 2 \mathrm{H}, \mathrm{CH}=\mathrm{N}), 7.95$ (dd, $J_{1}=7.6 \mathrm{~Hz}, J_{2}=1.7 \mathrm{~Hz}$, 2H, ArH), 7.41 (td, $\left.J_{1}=8.0 \mathrm{~Hz}, J_{2}=1.7 \mathrm{~Hz}, 2 \mathrm{H}, \mathrm{ArH}\right), 7.10-6.93$ (m, 4H, ArH), 4.39 (s, 4H, $\left.\mathrm{CH}_{2} \mathrm{O}\right), 3.60\left(\mathrm{t}, J=5.1 \mathrm{~Hz}, \mathrm{CH}_{2} \mathrm{~N}\right), 2.28\left(\mathrm{q}, J=5.12 \mathrm{H},-\mathrm{CH}_{2^{-}}\right) .{ }^{13} \mathrm{C}-\mathrm{NMR}(50 \mathrm{MHz}$, $\left.\mathrm{CDCl}_{3}\right) \delta=158.7,158.5,131.7,127.0,125.6,121.7,113.5,68.0,57.8,28.5$. ESI-MS: $309[\mathrm{M}+\mathrm{H}] .^{+}$

Synthesis of diimine $5:^{2}$ A mixture of $2.0 \mathrm{~g}$ of dialdehyde $2(7.05 \mathrm{mmol})$, in $400 \mathrm{~mL}$ MeOH and 1,3-diaminopropane, (0.52 g, $7.05 \mathrm{mmol})$ in $\mathrm{MeOH},(100 \mathrm{~mL})$, was stirred and refluxed for 3 hours. The solvent was removed at reduced pressure affording $2.19 \mathrm{~g}$, (95\%) of a pale yellow solid. mp 130-132 ${ }^{\circ} \mathrm{C}$ (Hexane-CHCl 3 ) (lit. $\left.{ }^{2} 128-130{ }^{\circ} \mathrm{C}\right) .{ }^{1} \mathrm{H}-$ NMR (200 MHz, $\left.\mathrm{CDCl}_{3}\right) \delta=8.68(\mathrm{~s}, 2 \mathrm{H}, \mathrm{CH}=\mathrm{N}), 7.96\left(\mathrm{dd}, J_{1}=7.5 \mathrm{~Hz}, J_{2}=1.8 \mathrm{~Hz}\right.$, 2H, ArH), 7.39 (dt, $\left.J_{1}=7.5 \mathrm{~Hz}, J_{2}=1.8 \mathrm{~Hz}, 2 \mathrm{H}, \mathrm{ArH}\right), 7.06-6.95$ (m, 4H, ArH), 4.32 (t, $J$ $\left.=5.1 \mathrm{~Hz}, 4 \mathrm{H}, \mathrm{CH}_{2} \mathrm{O}\right), 3.61\left(\mathrm{t}, J=5.1 \mathrm{~Hz}, \mathrm{CH}_{2} \mathrm{~N}\right), 2.33-2.21\left(\mathrm{~m}, 4 \mathrm{H},-\mathrm{CH}_{2}-\right) .{ }^{13} \mathrm{C}-\mathrm{NMR}$ $\left(50 \mathrm{MHz}, \mathrm{CDCl}_{3}\right) \delta=158.7,158.2,131.7,127.5,125.5,121.2,112.8,68.3,57.1,29.5$, 28.7. ESI-MS: $323.1[\mathrm{M}+\mathrm{H}]{ }^{+}$ 
Synthesis of diimine 6: A mixture of $1.0 \mathrm{~g}$ of dialdehyde 1 (3.7 mmol), in $200 \mathrm{~mL}$ $\mathrm{MeOH}$, and 1,4-diamine-2-butyne, ${ }^{3}$ (0.312 g, $3.75 \mathrm{mmol}$ ) in $100 \mathrm{~mL} \mathrm{MeOH}$, was stirred and refluxed for 3 hours. The solvent was removed at reduced pressure affording $1.18 \mathrm{~g}$, (97\%) of a pale yellow solid. mp. $155-157^{\circ} \mathrm{C} .{ }^{1} \mathrm{H}-\mathrm{NMR}\left(300 \mathrm{MHz}, \mathrm{CDCl}_{3}\right) \delta=9.38(\mathrm{~s}$, 2H, CH=N), 7.92 (d, J= 7.5 Hz, 2H, ArH), 7.40 (t, $J=7.2 \mathrm{~Hz}, 2 \mathrm{H}, \mathrm{ArH}), 7.08-7.00$ (m, $4 \mathrm{H}, \mathrm{ArH}), 4.73\left(\mathrm{~s}, 4 \mathrm{H}, \mathrm{CH}_{2} \mathrm{~N}\right), 4.41\left(\mathrm{~s}, 4 \mathrm{H}, \mathrm{CH}_{2} \mathrm{O}\right) .{ }^{13} \mathrm{C}-\mathrm{NMR}\left(75 \mathrm{MHz}, \mathrm{CDCl}_{3}\right) \delta=$ 157.8, 157.5, 131.8, 127.6, 126.1, 122.0, 113.5, 83.1, 67.4, 46.7. Anal. Calcd. for $\mathrm{C}_{20} \mathrm{H}_{18} \mathrm{~N}_{2} \mathrm{O}_{2}$ : C, 75.45; H, 5.70. Found: C, 75.76; H, 5.82.

Synthesis of diimine 7: A mixture of $0.5 \mathrm{~g}$ of dialdehyde $3(1.3 \mathrm{mmol})$ in $150 \mathrm{~mL}$ EtOH and 1,3-diaminopropane, (97 mg, $1.3 \mathrm{mmol}$ ) in $50 \mathrm{~mL}$ EtOH was stirred and refluxed for 17 hours. The solvent was removed at reduced pressure affording $0.55 \mathrm{~g}$, (92\%) of a brown yellow solid. mp.137-140 ${ }^{\circ}$ C. ${ }^{1} \mathrm{H}-\mathrm{NMR}\left(300 \mathrm{MHz}, \mathrm{CDCl}_{3}\right) \delta=9.12$ (d, $J=8.8 \mathrm{~Hz}, 2 \mathrm{H}, \mathrm{ArH}), 9.07$ (s, 2H, CH=N), 7.89 (d, $J=9.0 \mathrm{~Hz}, 2 \mathrm{H}, \mathrm{ArH}), 7.78$ (d, $J$ = 8.1 Hz, 2H, ArH), 7.58-7.50 (m, 2H, ArH), 7.44-7.36 (m, 2H, ArH), 7.31 (d, J= 9.0 $\mathrm{Hz}, 2 \mathrm{H}, \mathrm{ArH}), 4.46$ (t, $\left.J=5.4 \mathrm{~Hz}, 4 \mathrm{H}, \mathrm{CH}_{2} \mathrm{O}\right), 3.81\left(\mathrm{t}, J=4.8 \mathrm{~Hz} 4 \mathrm{H}, \mathrm{CH}_{2} \mathrm{~N}\right), 2.45-2.35$ (m, 2H, $\mathrm{CH}_{2}$ ), 2.31(q, $\left.J=5.4 \mathrm{~Hz}, 2 \mathrm{H}, \mathrm{CH}_{2}\right) \cdot{ }^{13} \mathrm{C}-\mathrm{NMR}\left(75 \mathrm{MHz}, \mathrm{CDCl}_{3}\right) \delta=160.2$, 157.3, 132.2, 132.1, 129.8, 128.0, 127.9, 125.9, 124.4, 120.0, 116.0, 68.5, 57.9, 29.8, 29.5. Anal. Calcd. for $\mathrm{C}_{28} \mathrm{H}_{26} \mathrm{~N}_{2} \mathrm{O}_{2}$ : C, 75.45; H, 5.70. Found: 75.37; H, 5.86. 


\section{X-Ray Diffraction Analysis:}

The data were collected on a SMART CCD-BRUKER diffractometer, with graphitemonochromated Mo-K $\alpha$ radiation $(\lambda=0.71073 \AA)$ operating at $50 \mathrm{kV}$ and $25 \mathrm{~mA}$.

Crystal data: (8) $\mathrm{C}_{35} \mathrm{H}_{32} \mathrm{~N}_{2} \mathrm{O}_{6}, \mathrm{M}=576.63$, monoclinic, $\mathrm{P} 2{ }_{1} / \mathrm{n}, \mathrm{a}=21.667(2) \AA$, $\mathrm{b}=$ 9.9002(7) $\AA, \quad c=29.684(2) \AA, \quad \beta=110.114(1), V=5979.0(7) \AA^{3}, Z=8$, Dc $=$ $1.281 \mathrm{Mg} / \mathrm{m}^{3}, \mathrm{~F}(000)=2432, \mu=0.088 \mathrm{~mm}^{-1}$. The intensity data were collected over a hemisphere of the reciprocal space by combination of three exposure sets. Each exposure of 20 s covered 0.3 in $\omega$. The refinement was done by full matrix least-squares procedures on F2(SHELXTL version 5.1). The non-hydrogen atoms were refined anisotropically. The hydrogen atoms were included in calculated positions and refined as riding on the respective carbon bonded atoms. Final $\mathrm{R}(\mathrm{Rw})$ value was 4.39 (14.02).

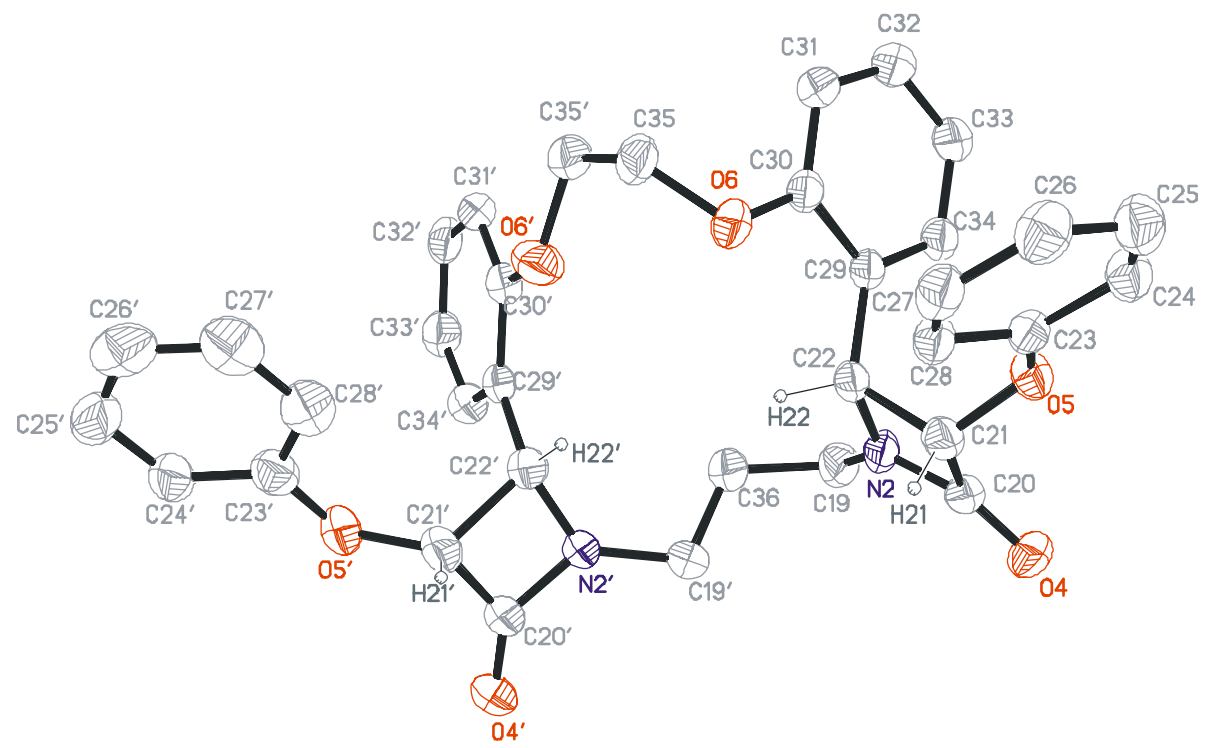

Crystal data of the $\mathrm{Co}_{2} \mathrm{CO}_{6}$ complex of 12: $\mathrm{C}_{42} \mathrm{H}_{30} \mathrm{Co}_{2} \mathrm{~N}_{2} \mathrm{O}_{12}, \mathrm{M}=872.54$, monoclinic, $\mathrm{P} 2{ }_{1} / \mathrm{c}, \mathrm{a}=20.785(2) \AA, \mathrm{b}=11.9521(9) \AA, \mathrm{c}=16.836(1) \AA, \beta=108.458(2), \mathrm{V}=$ 3967.3(5) $\AA^{3}, \mathrm{Z}=4, \mathrm{Dc}=1.461 \mathrm{Mg} / \mathrm{m}^{3}, \mathrm{~F}(000)=1784, \mu=0.902 \mathrm{~mm}^{-1}$. The hydrogen atoms were included in calculated positions and refined as riding on the respective carbon bonded atoms with the exception of H3, H4, H3' and H4' which were located in 
a Fourier difference and their coordinates were refined. The final $\mathrm{R}(\mathrm{Rw})$ value was 4.62 (11.72).

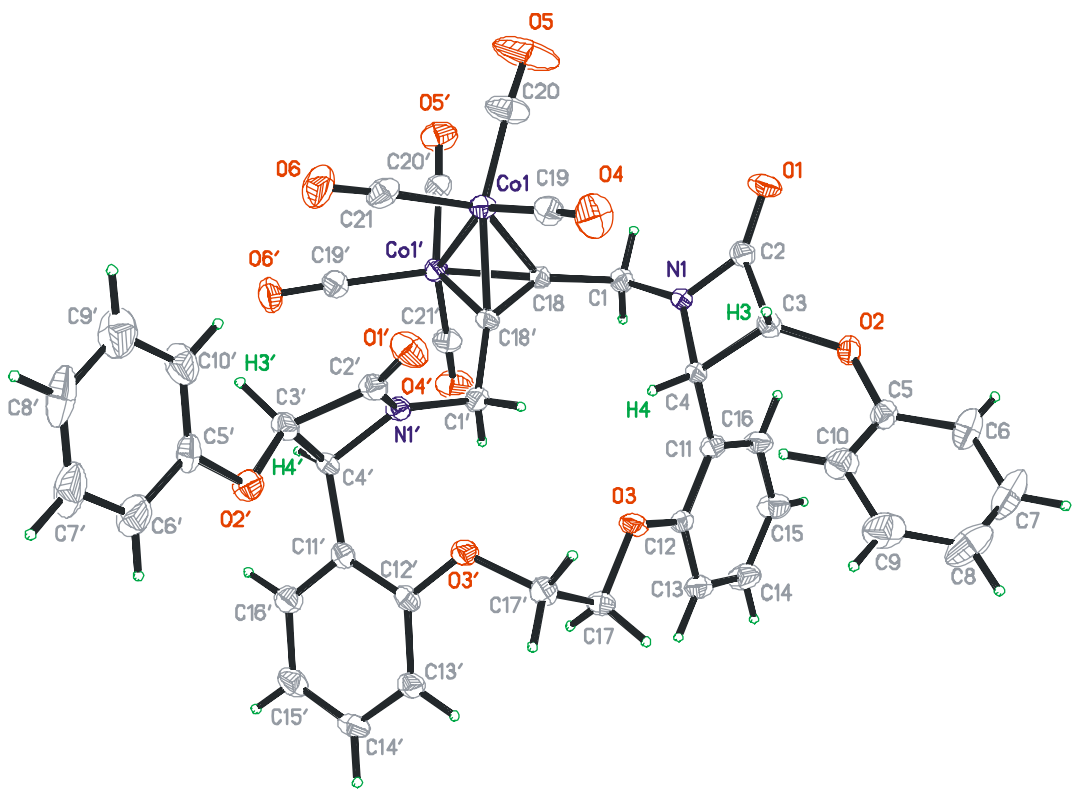

\section{REFERENCES}

(1) Simion, C.; Simion, A.; Mitoma, Y.; Nagashima, S.; Kawaji, T.; Hasimoto, I.; Tashiro, M. Heterocycles 2000, 53, 2459.

(2) a) Simion, A.; Simion, C.; Kanda, T.; Nagashima, S.; Mitoma, T.; Yamada, T.; Mimura, K.; Tashiro, M. J. Chem. Soc., Perkin Trans. I 2001, 2071. b) Armstrong, L. G.; Lindoy, L. F. Inorg. Chem. 1975, 14, 1322.

(3) Johnson, A. J. Chem. Soc., 1946, 1009. 
S-6

Dialdehyde 3

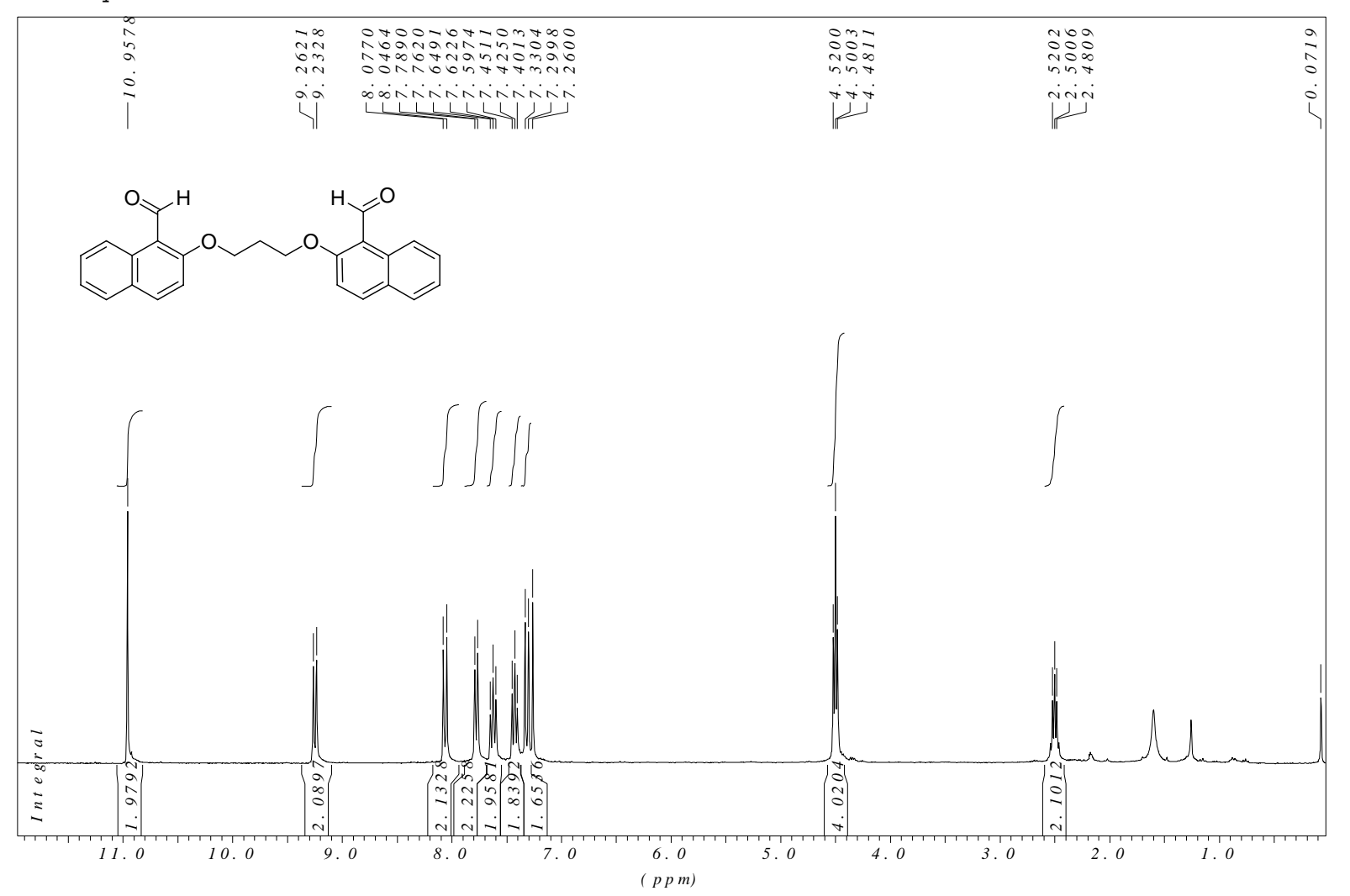

Dialdehyde 3

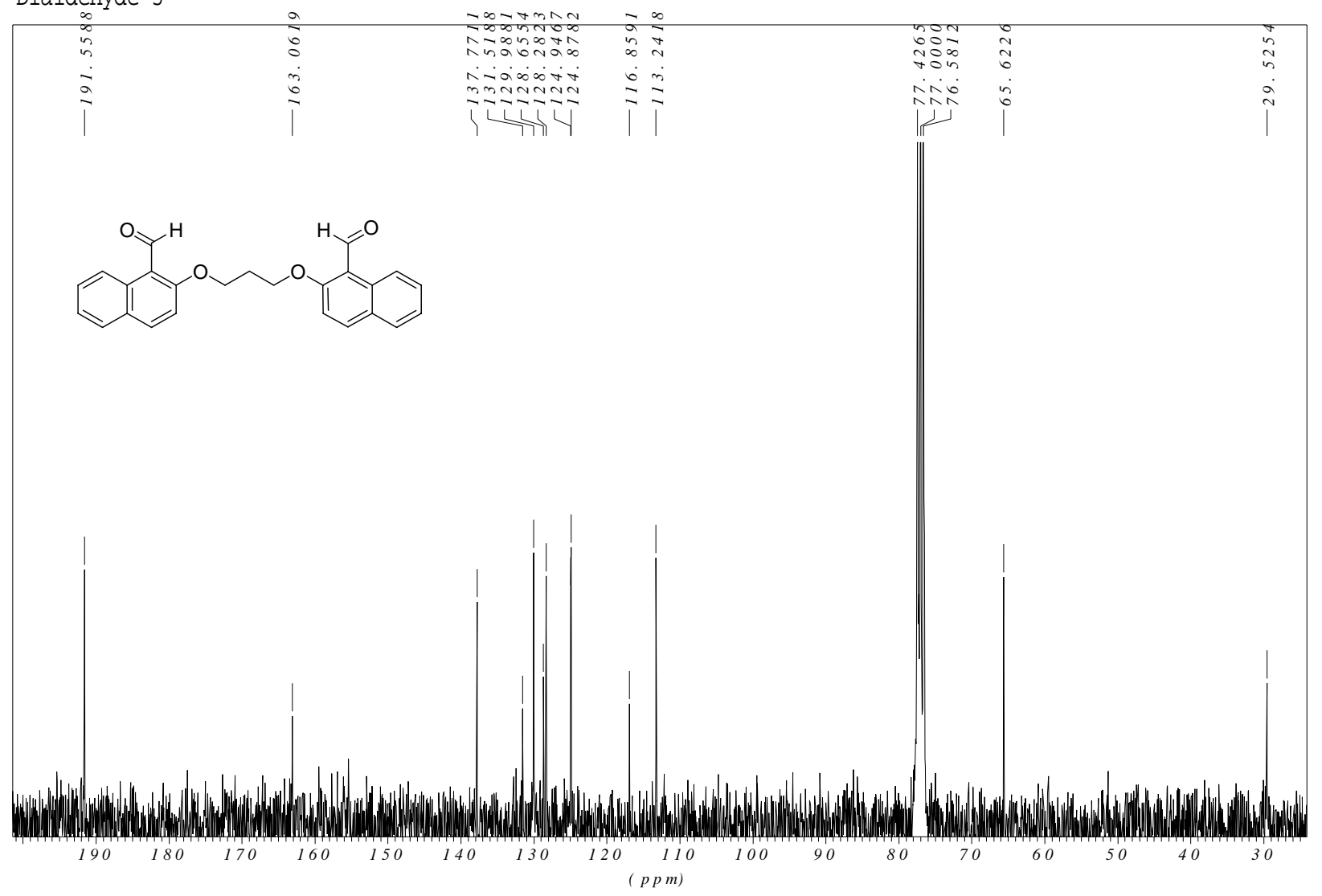


S-7

Diimine 4
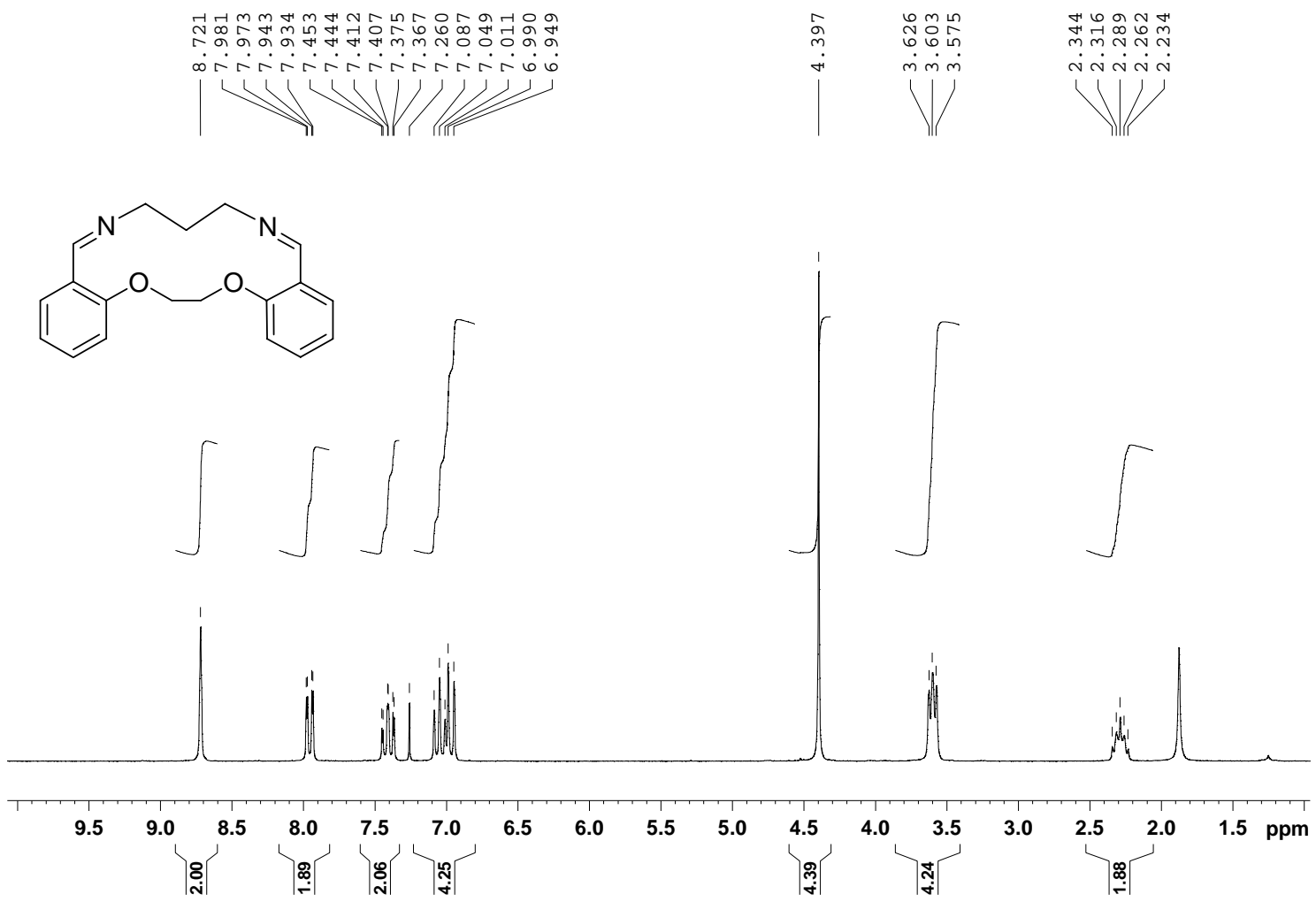

Diimine 4
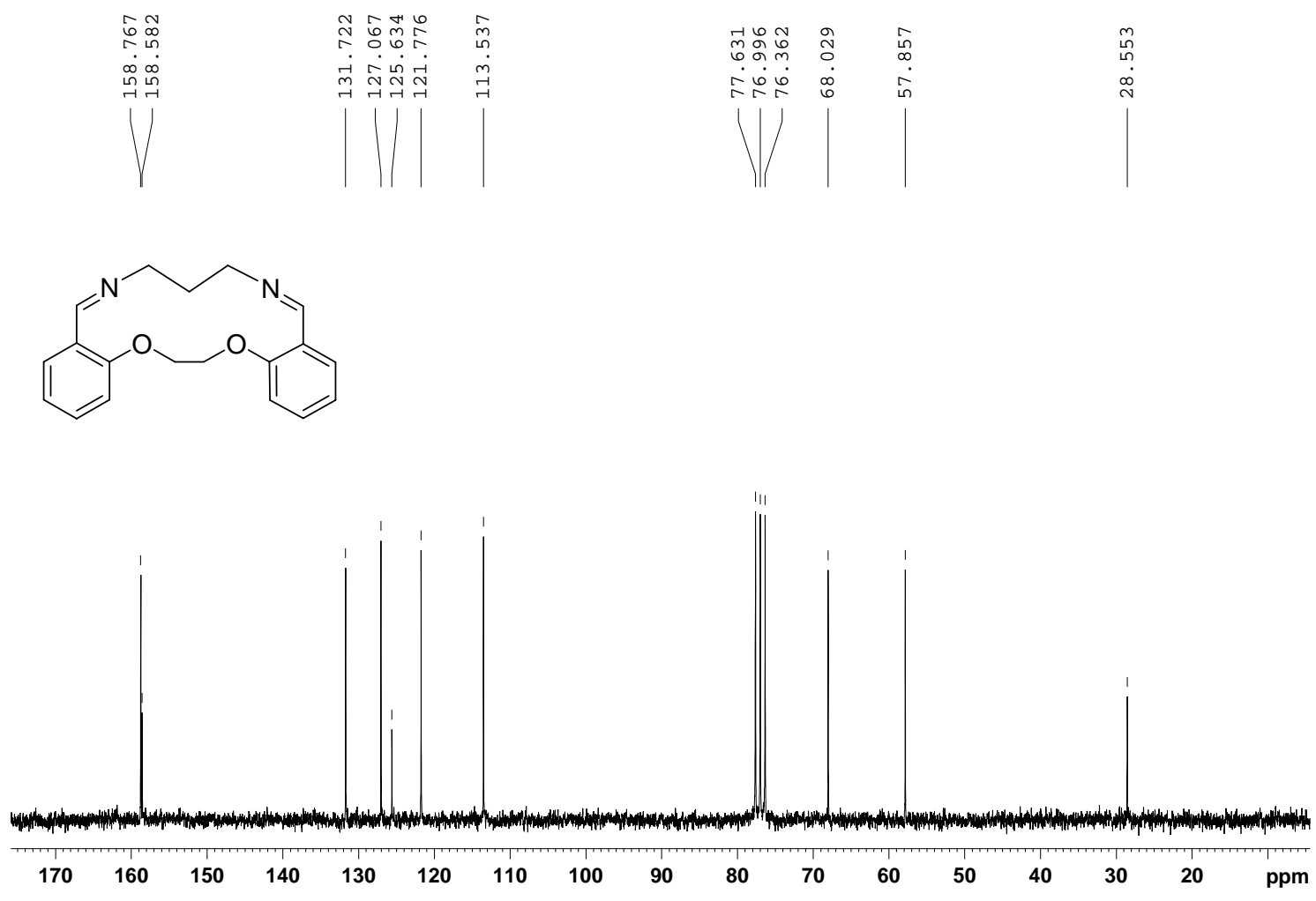
Diimine 5
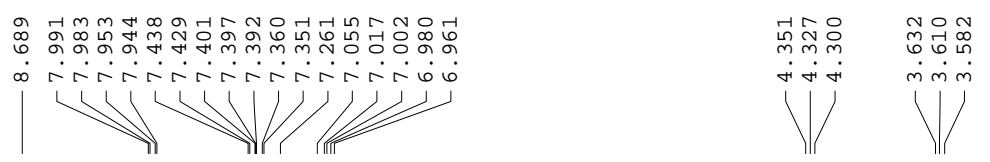

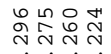

กั่

VI<smiles>C1=NCCC/N=C/c2ccccc2OCCCOc2ccccc2/C=N\CC1</smiles>

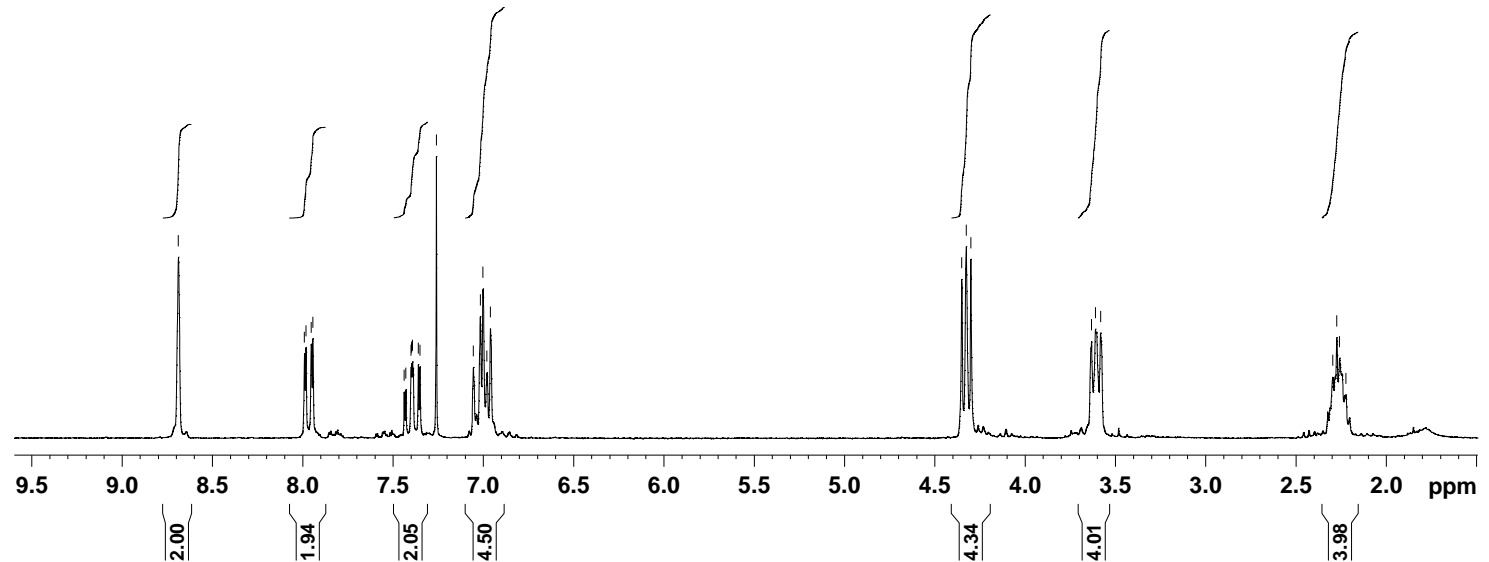

Diimine 5

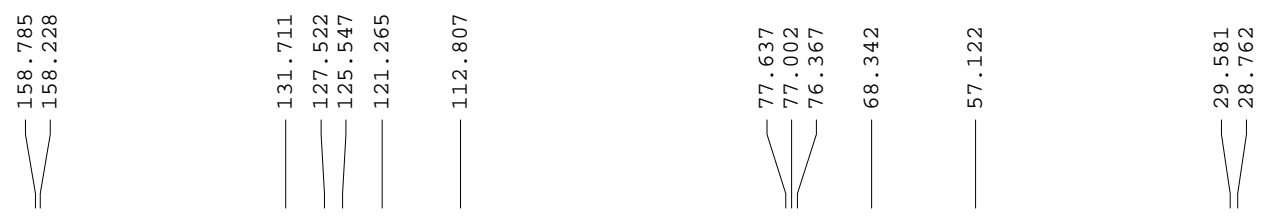<smiles>C1=N\CCC/N=C/c2ccccc2OCCCOc2ccccc2/1</smiles>

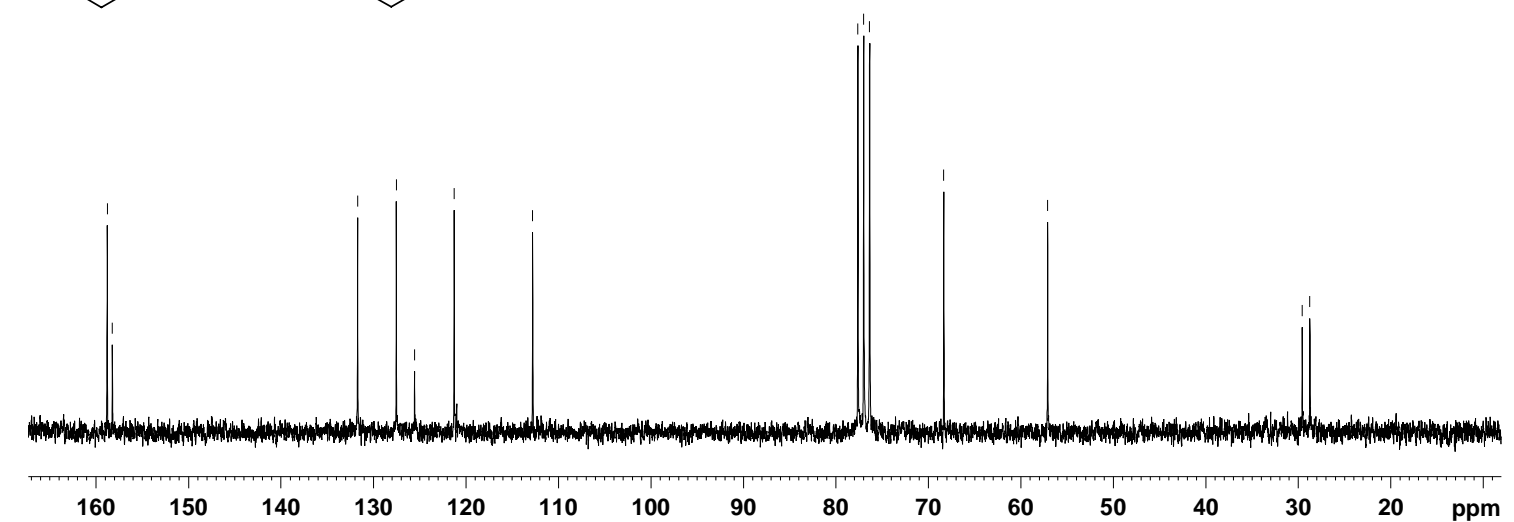


S-9
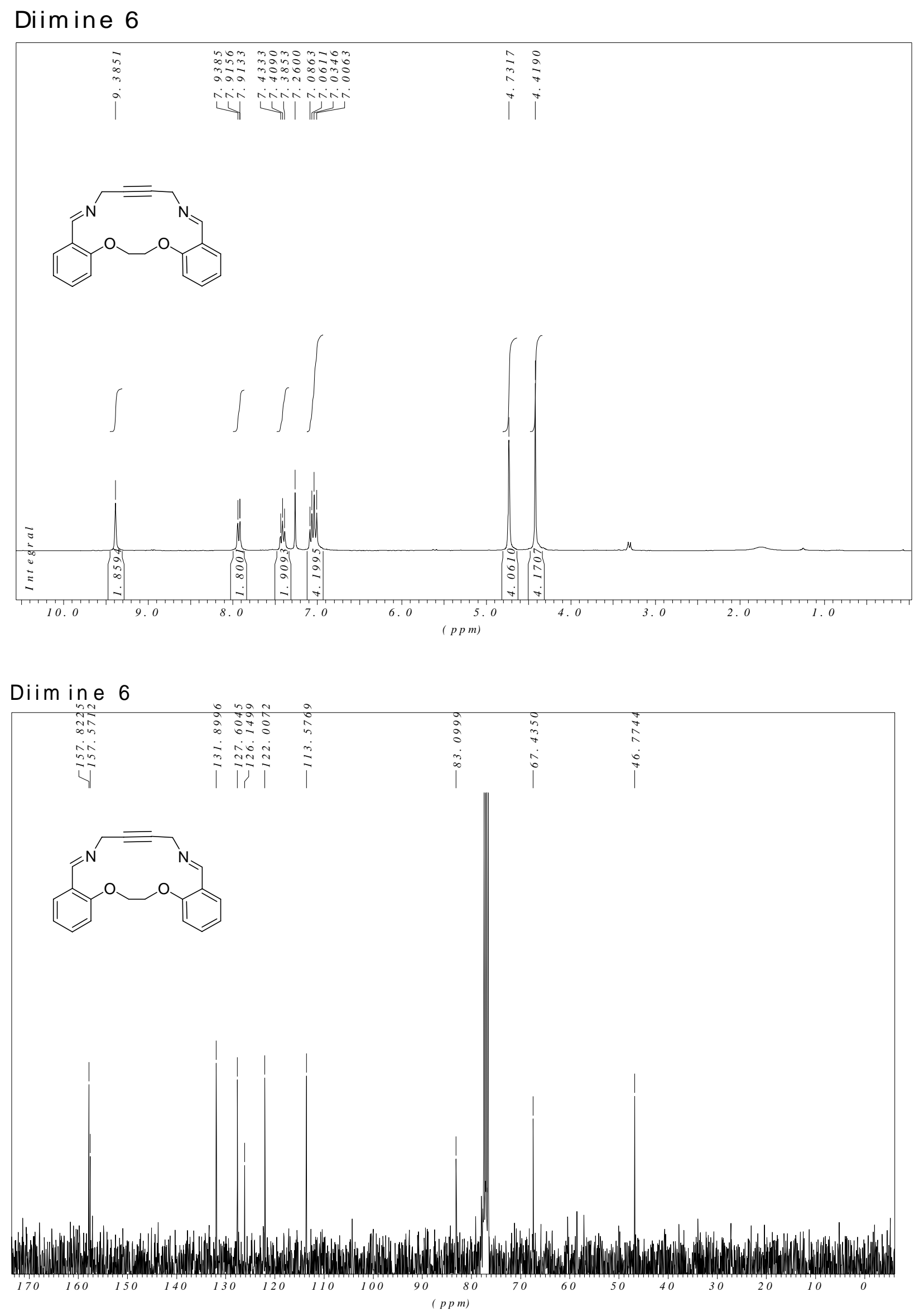

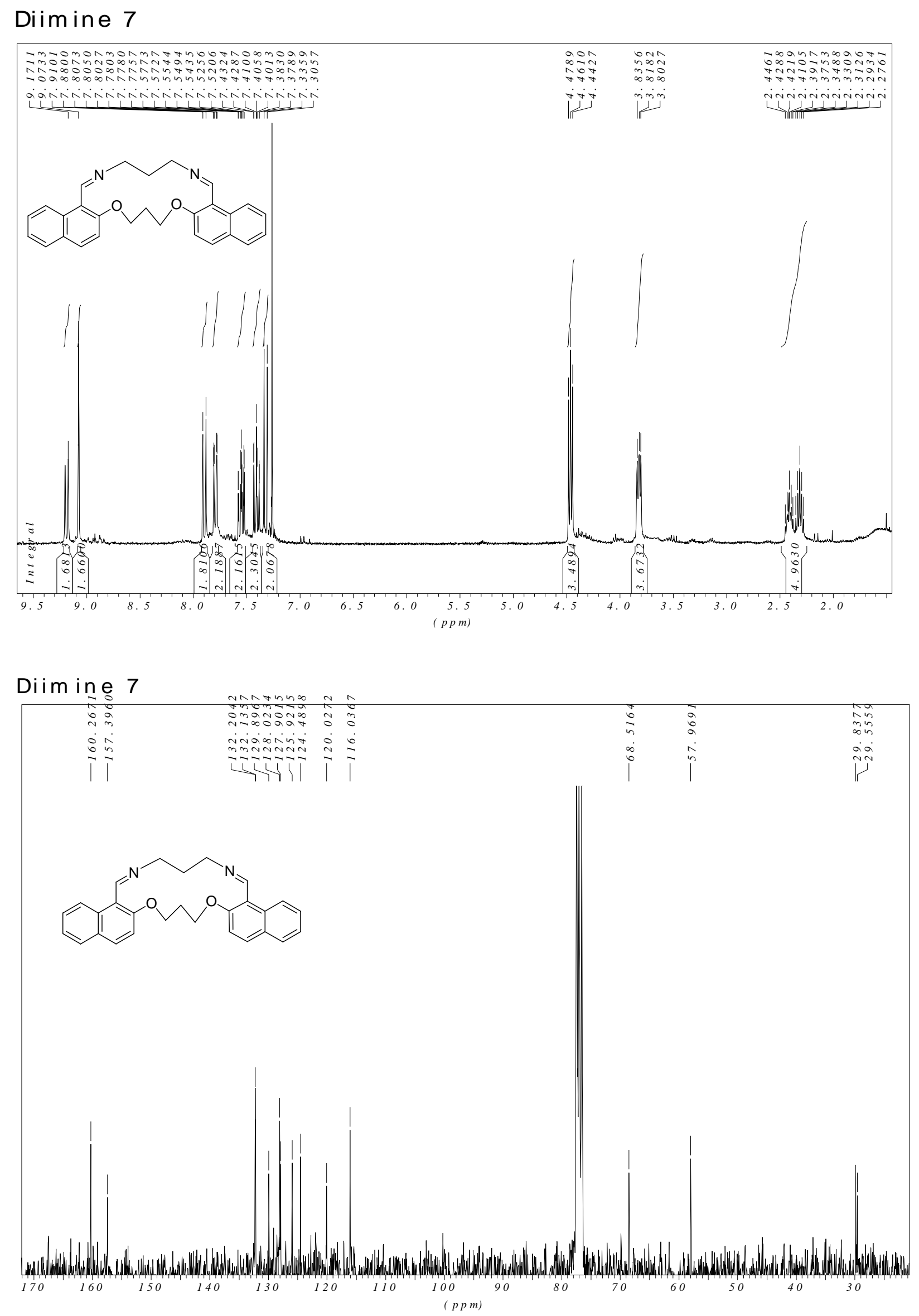


\section{b-Lactam 8}

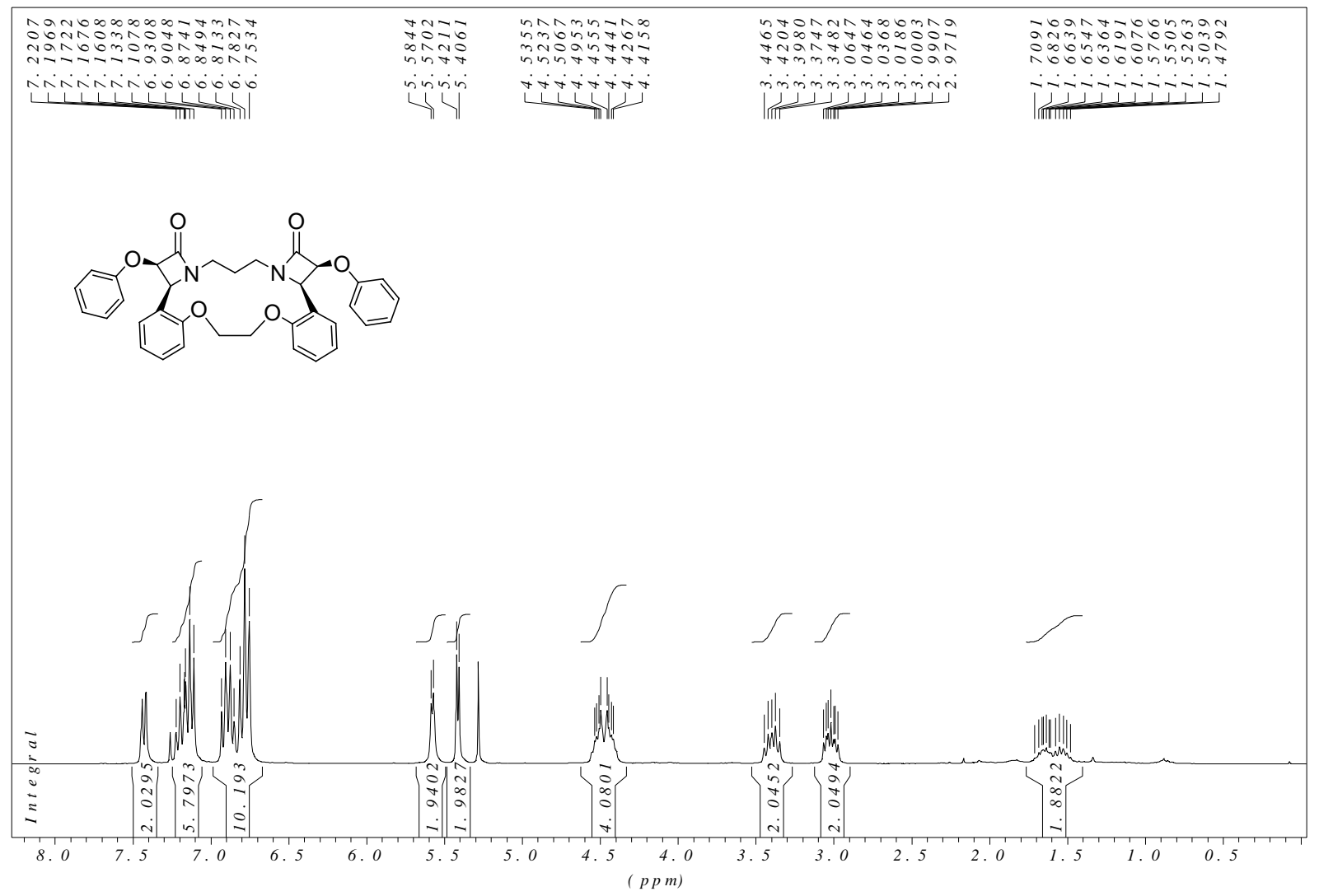

b-Lactam 8

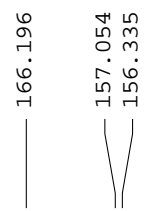

12

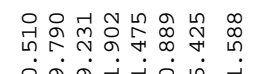

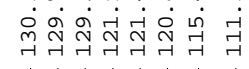
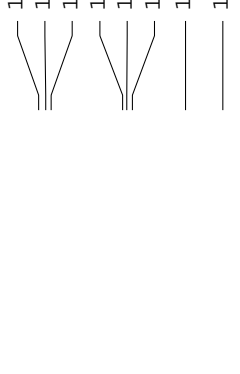
S-12

b-Lactam 9

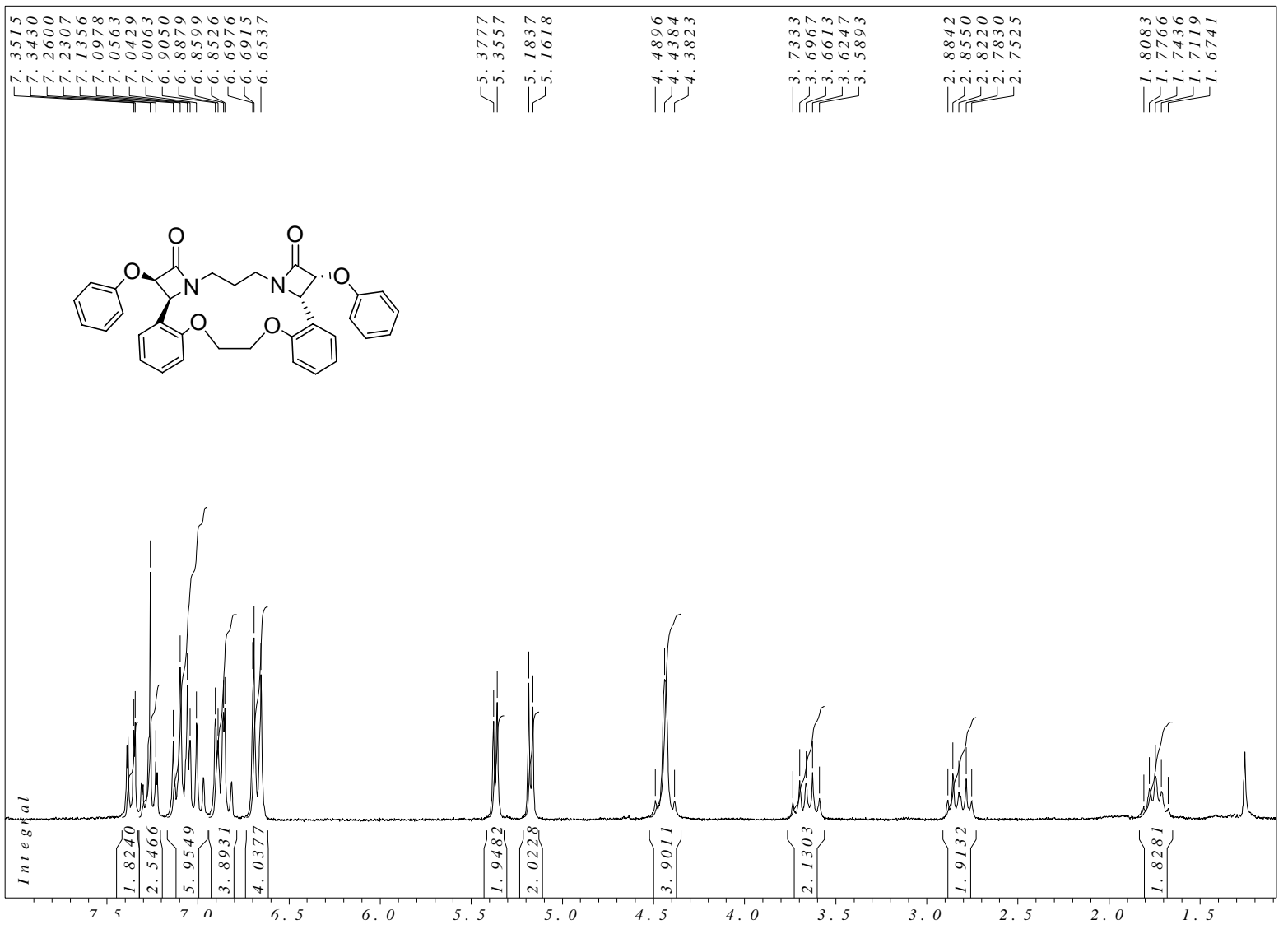

b-Lactam 9

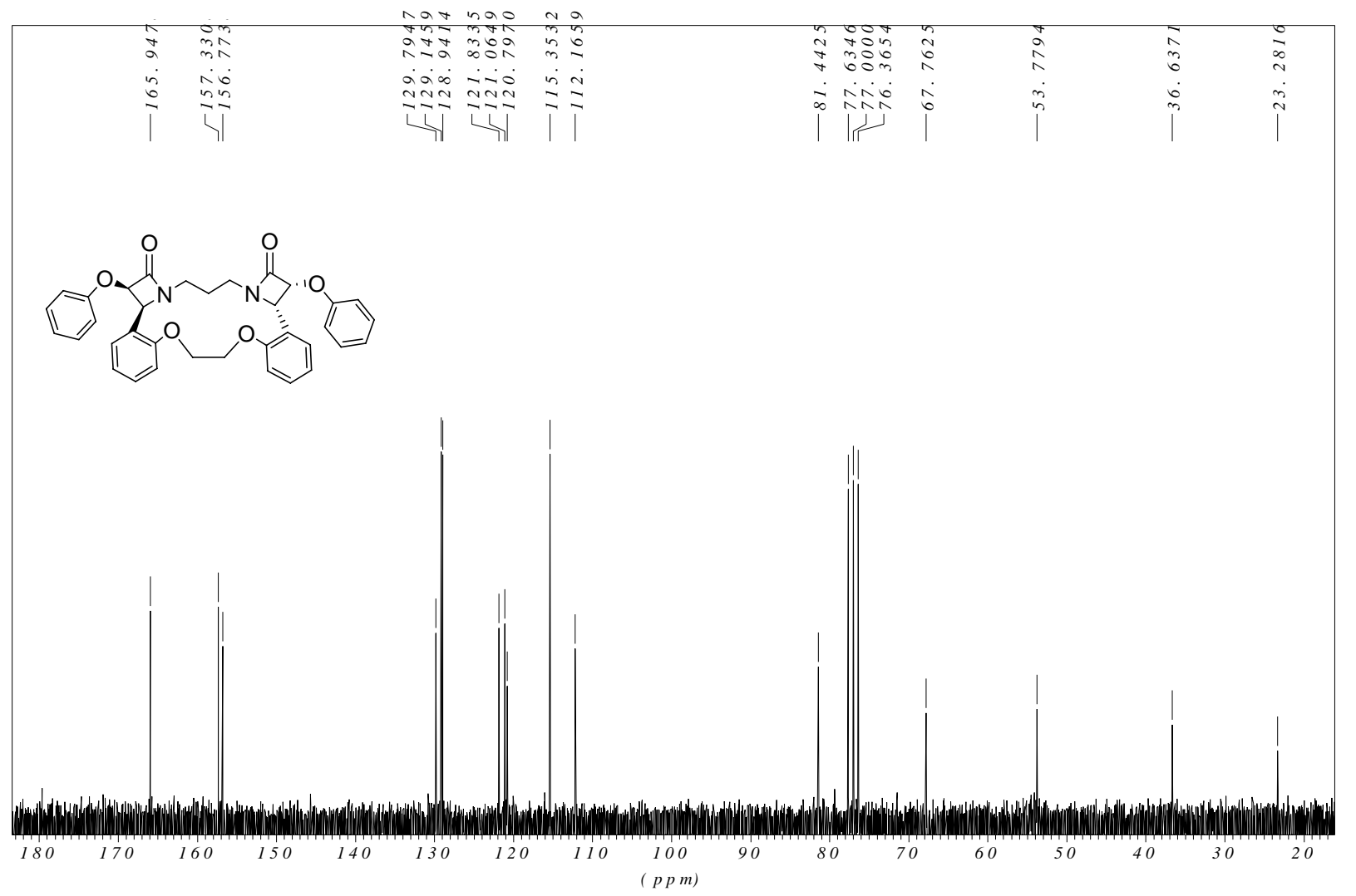


b-Lactam 10

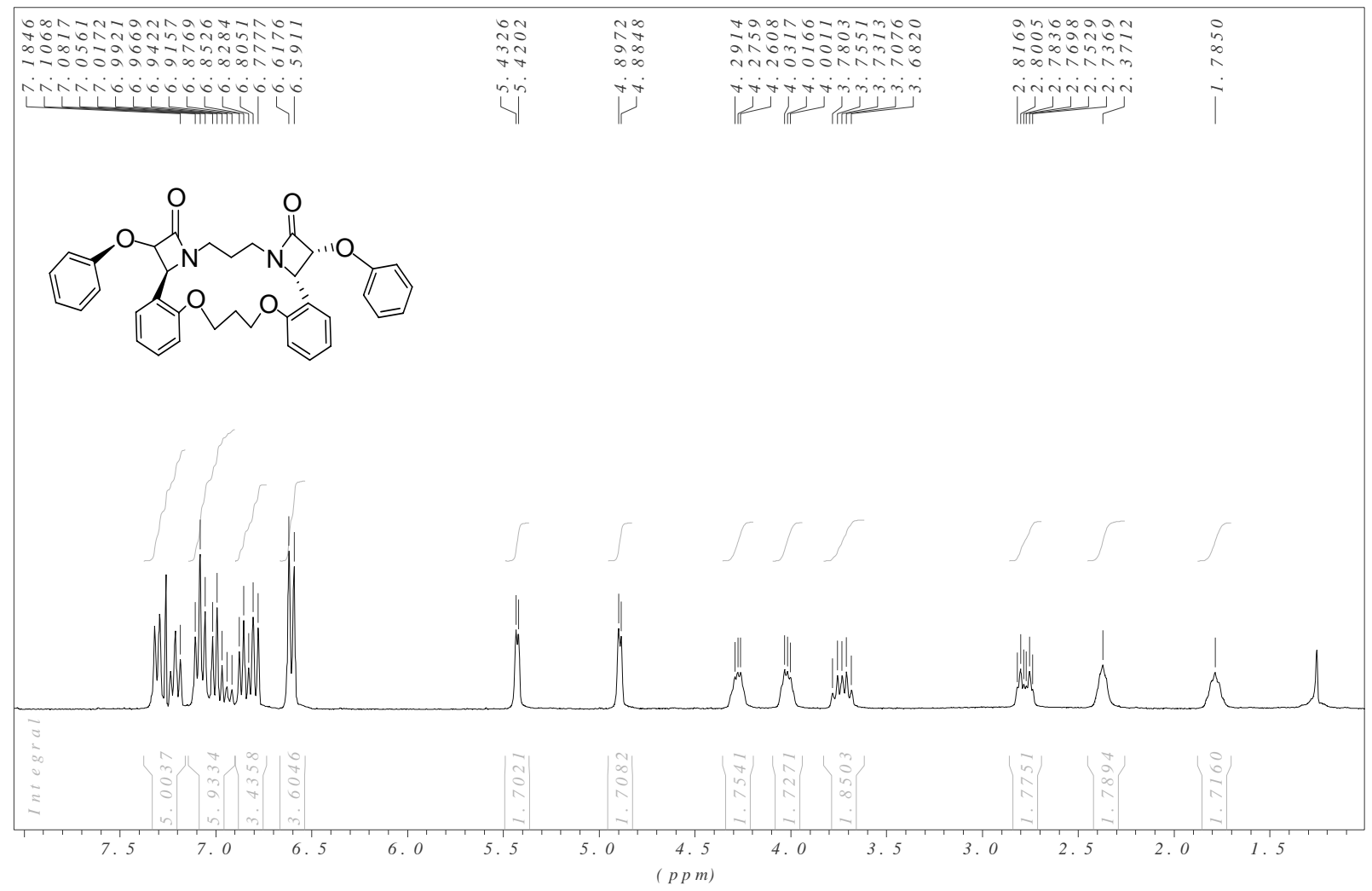

b-Lactam 10

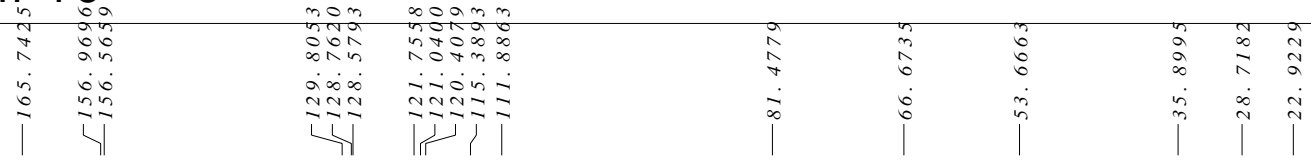<smiles>O=C1[C@H](Oc2ccccc2)[C@H](c2ccccc2)N1CCCOc1ccccc1OCCCOc1ccccc1</smiles>

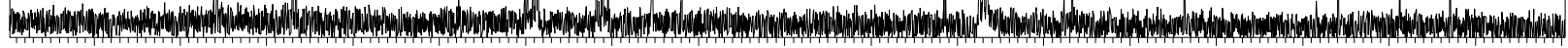

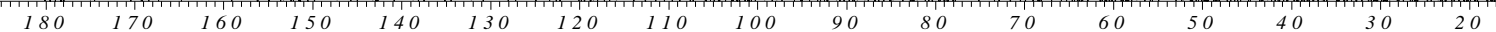
$(p p m)$ 


\section{b-Lactam 11}
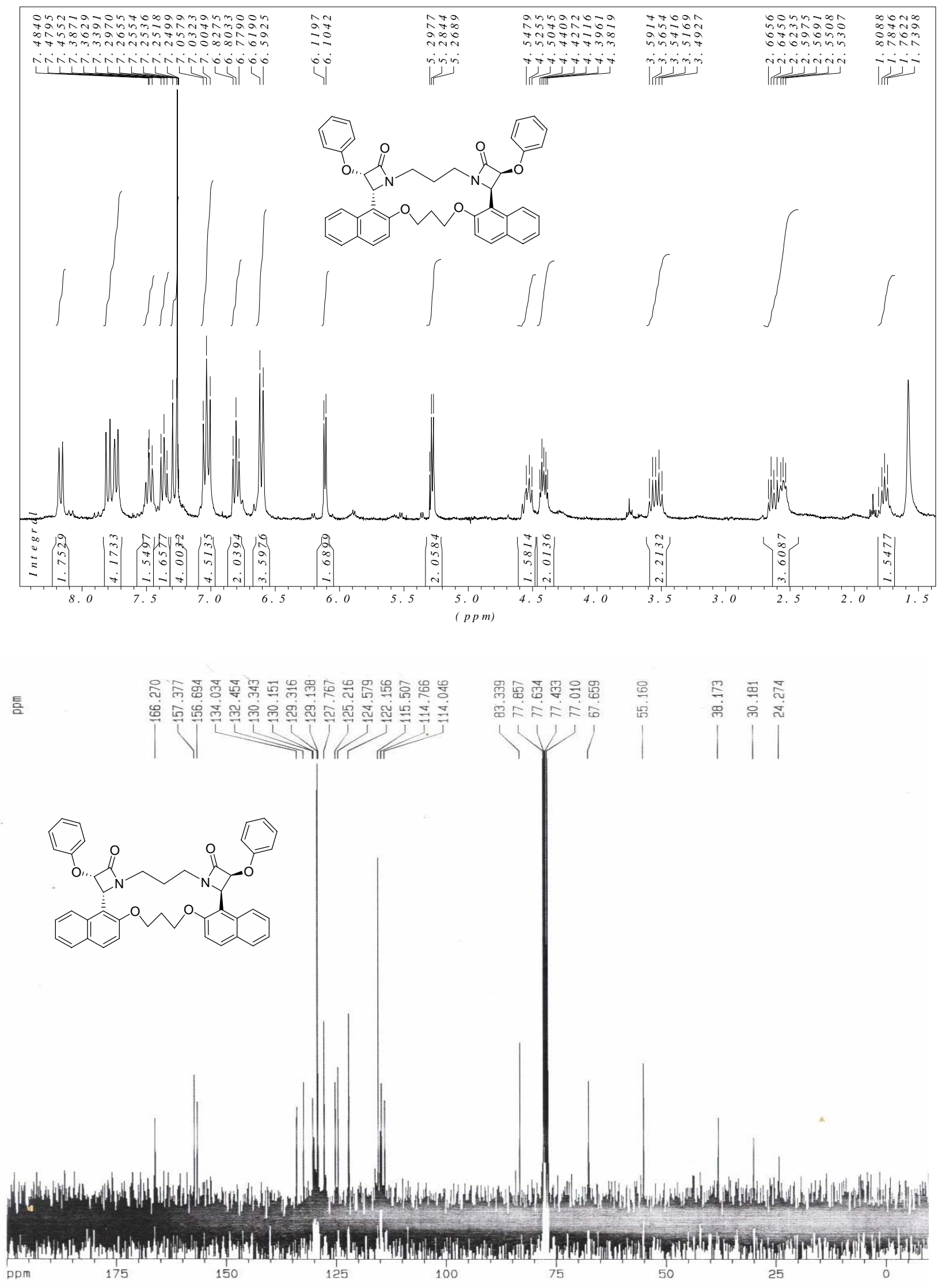
S-15
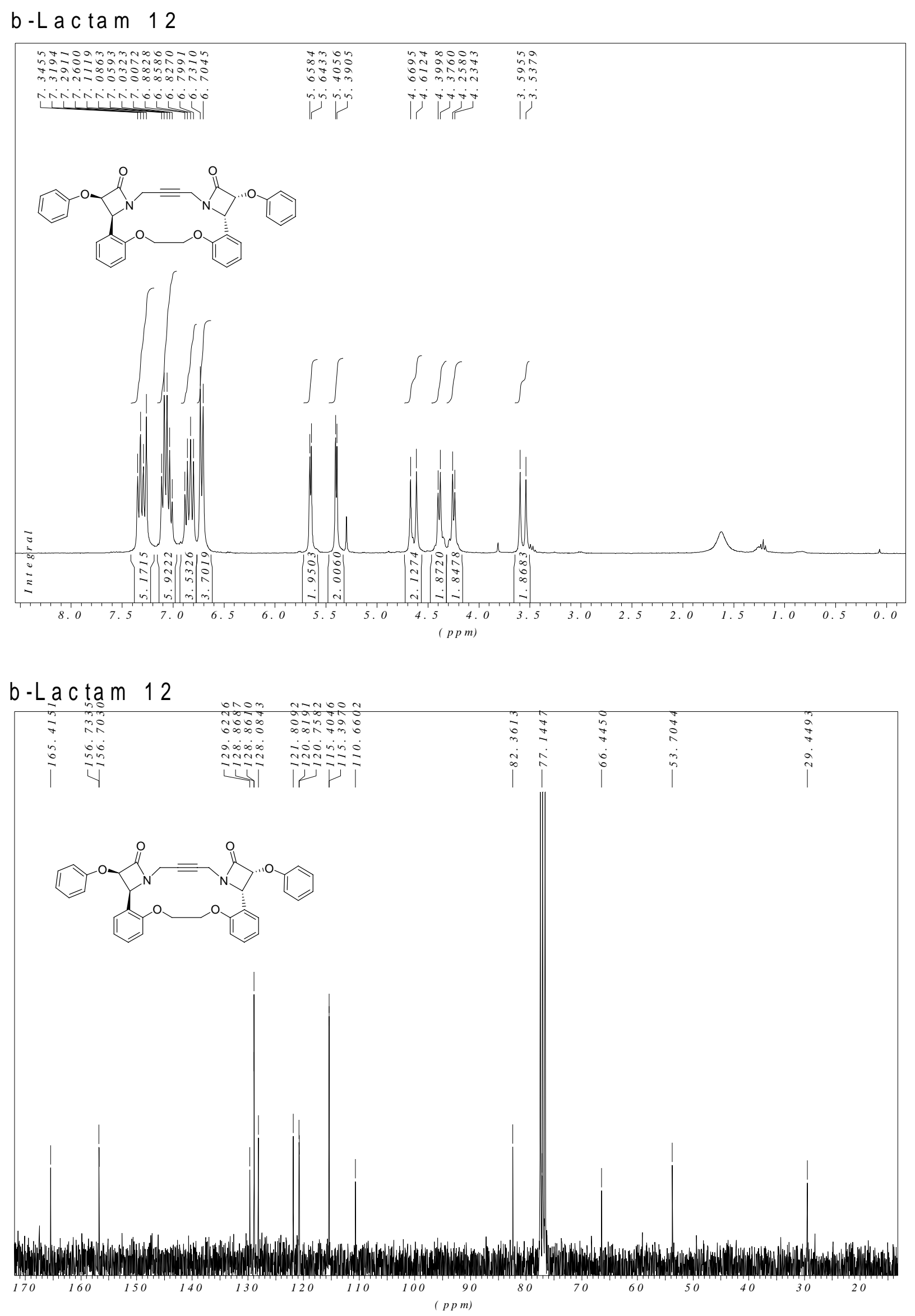
S-16
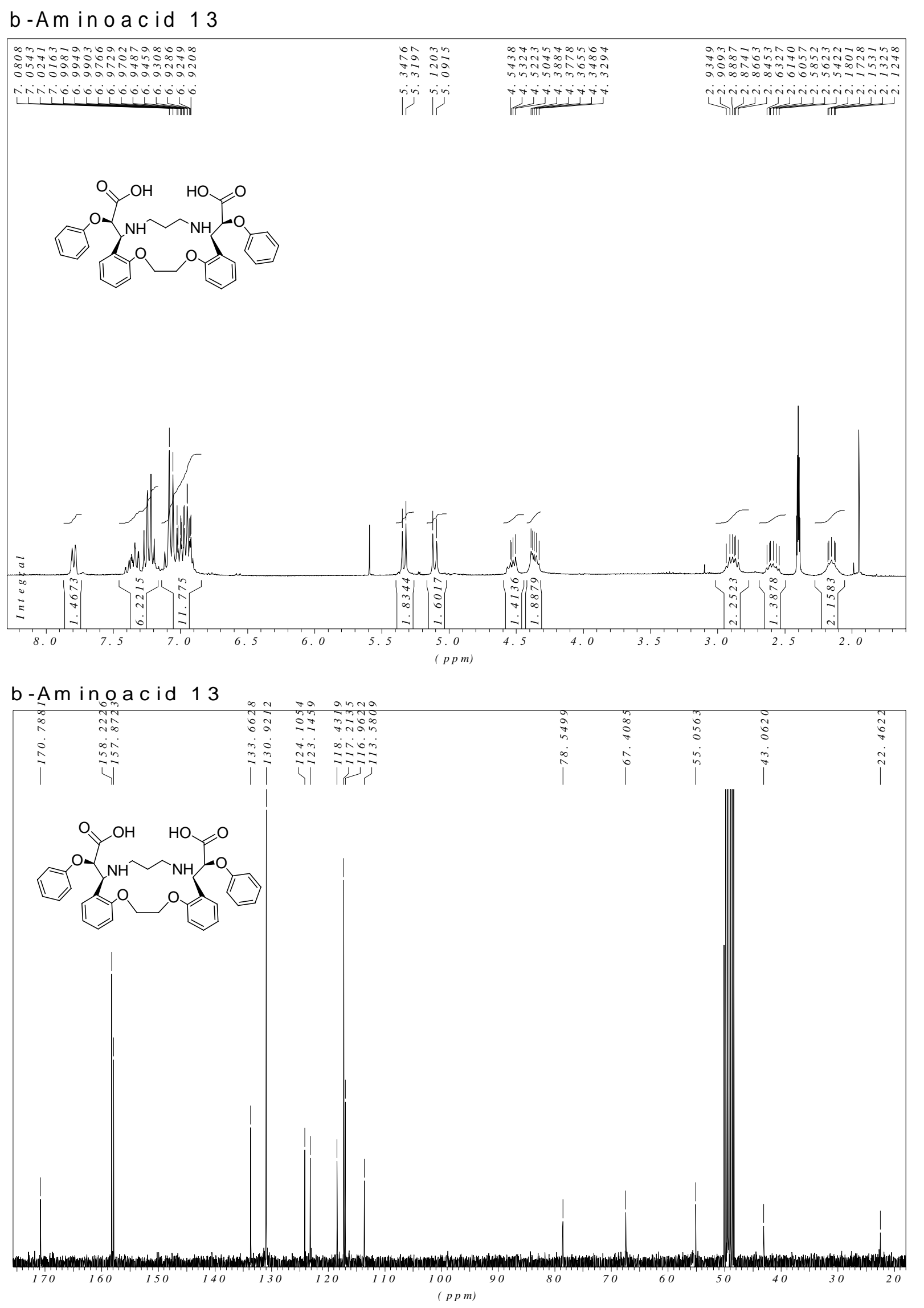
b-Am inoacid 14

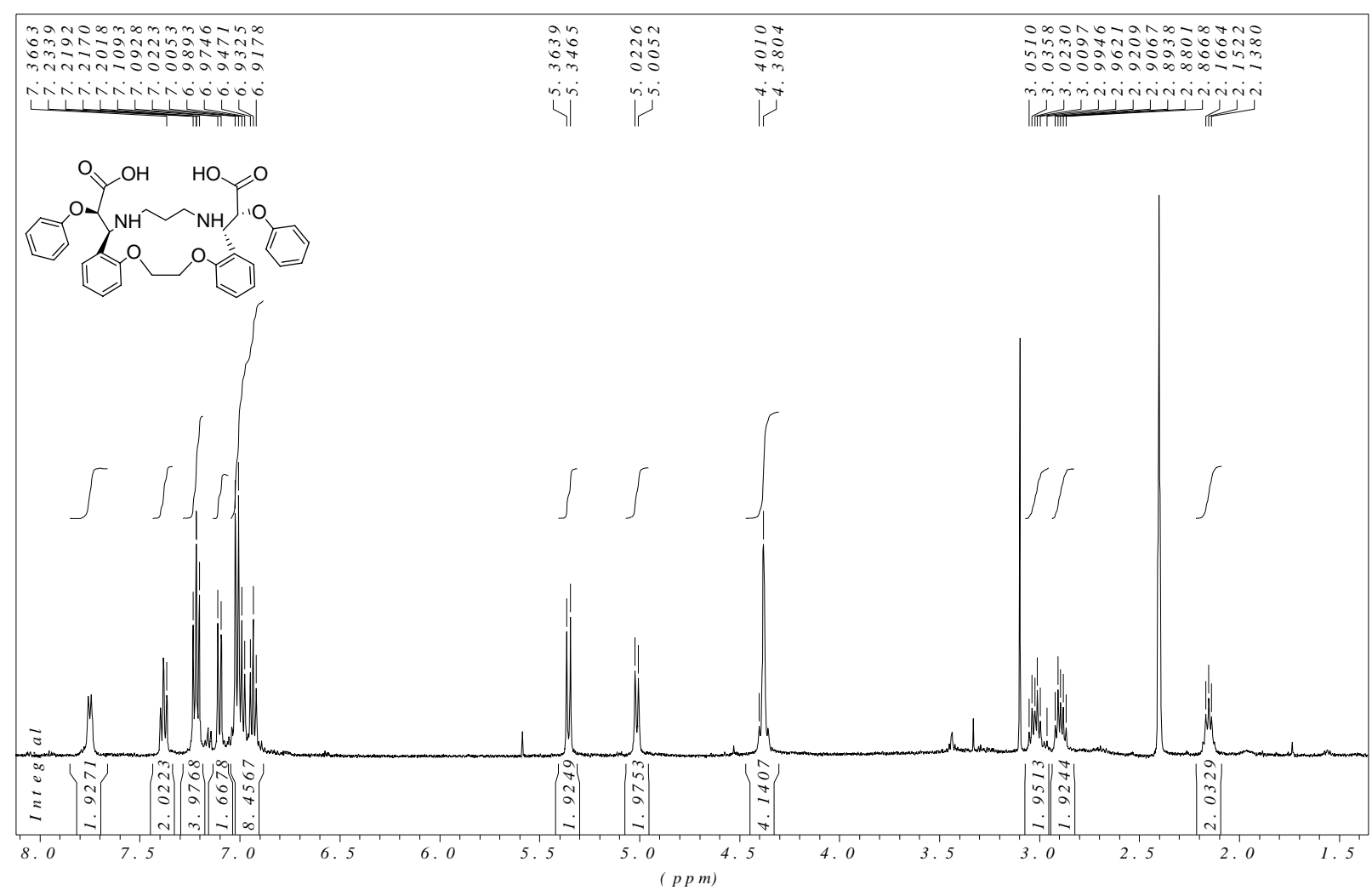

b-Am in 0 a c id 14

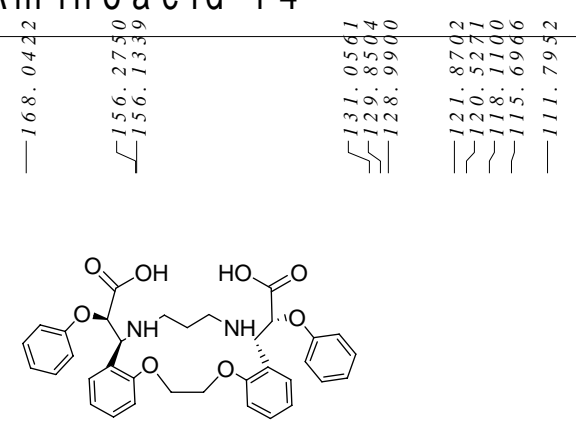

(1)

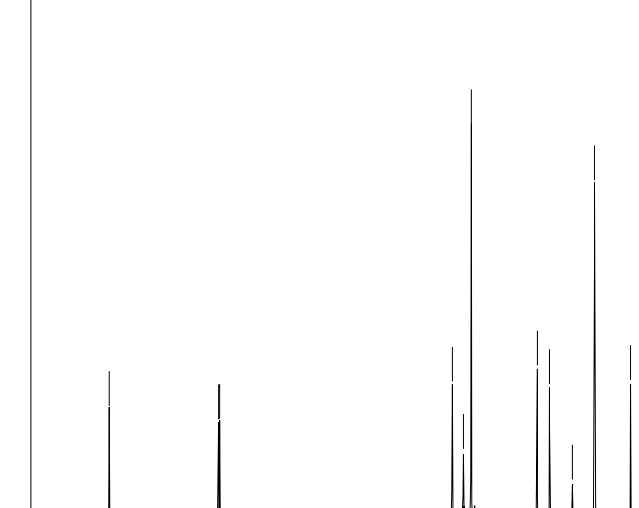

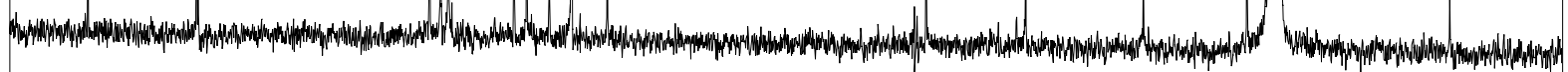

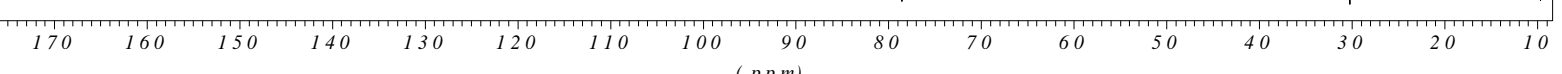


b-Amin 0 acid 15

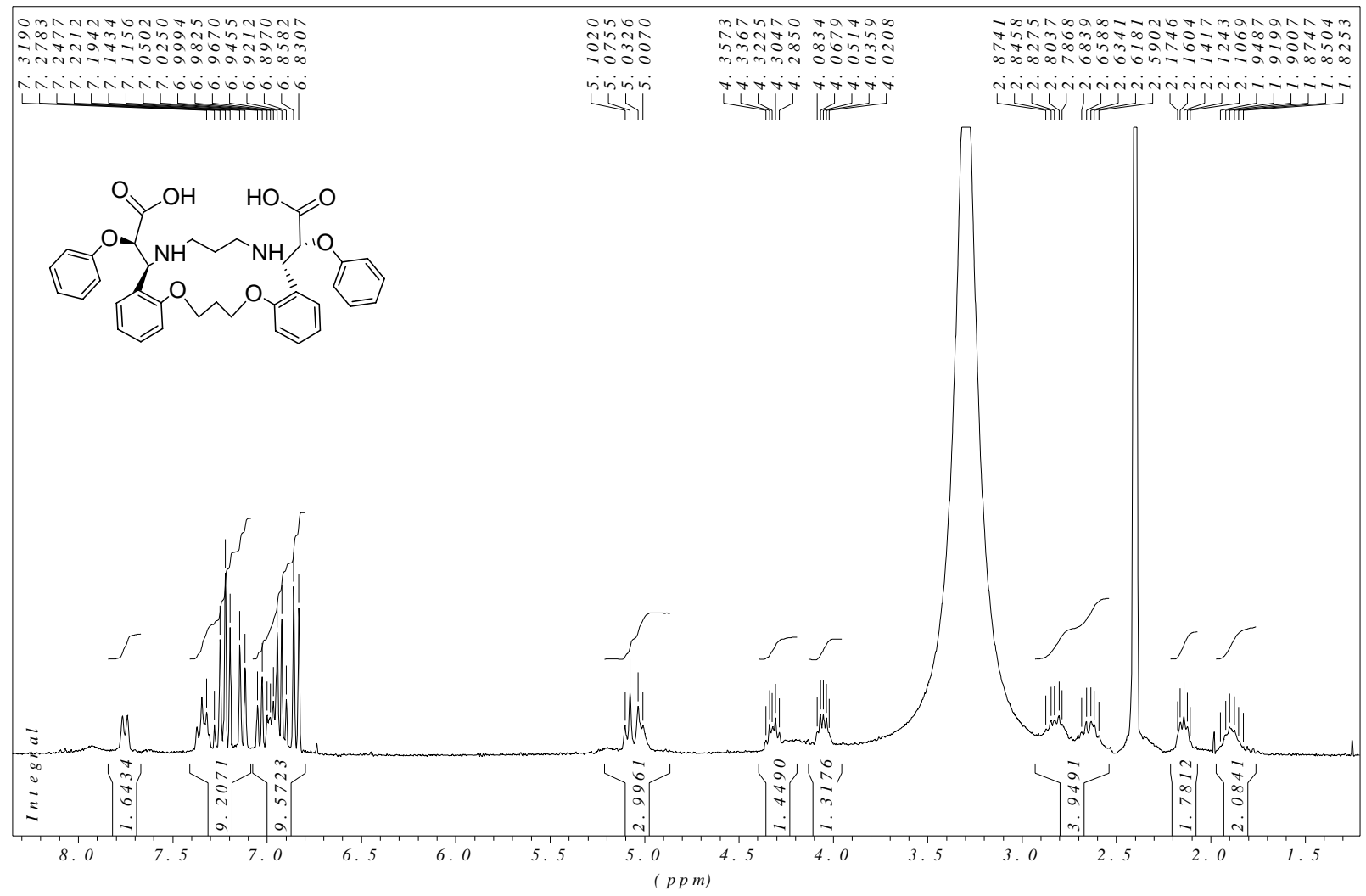

b-Am inoacid 15

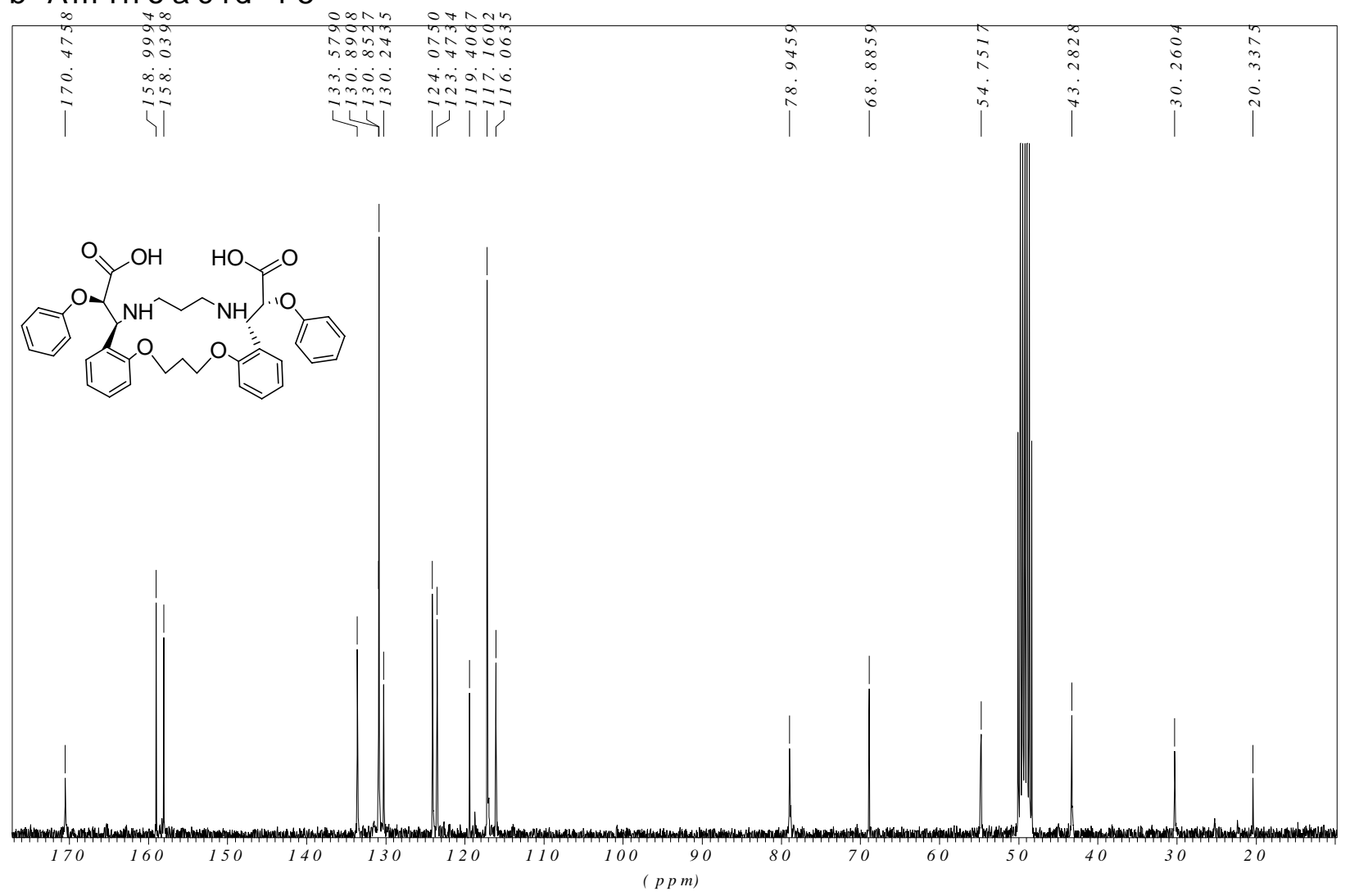


S-19

b-Aminoacid 16

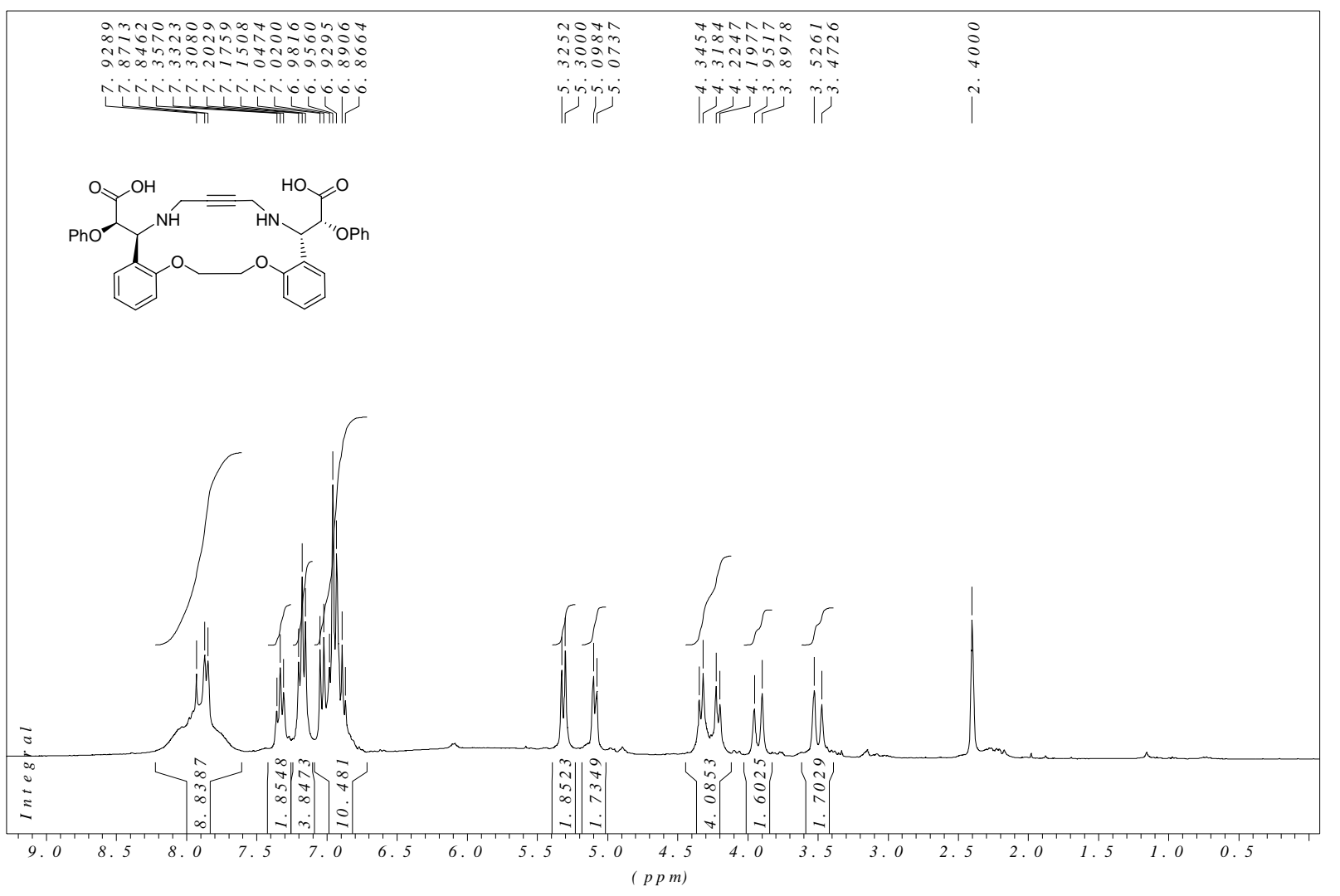

b-Am inoacid 16

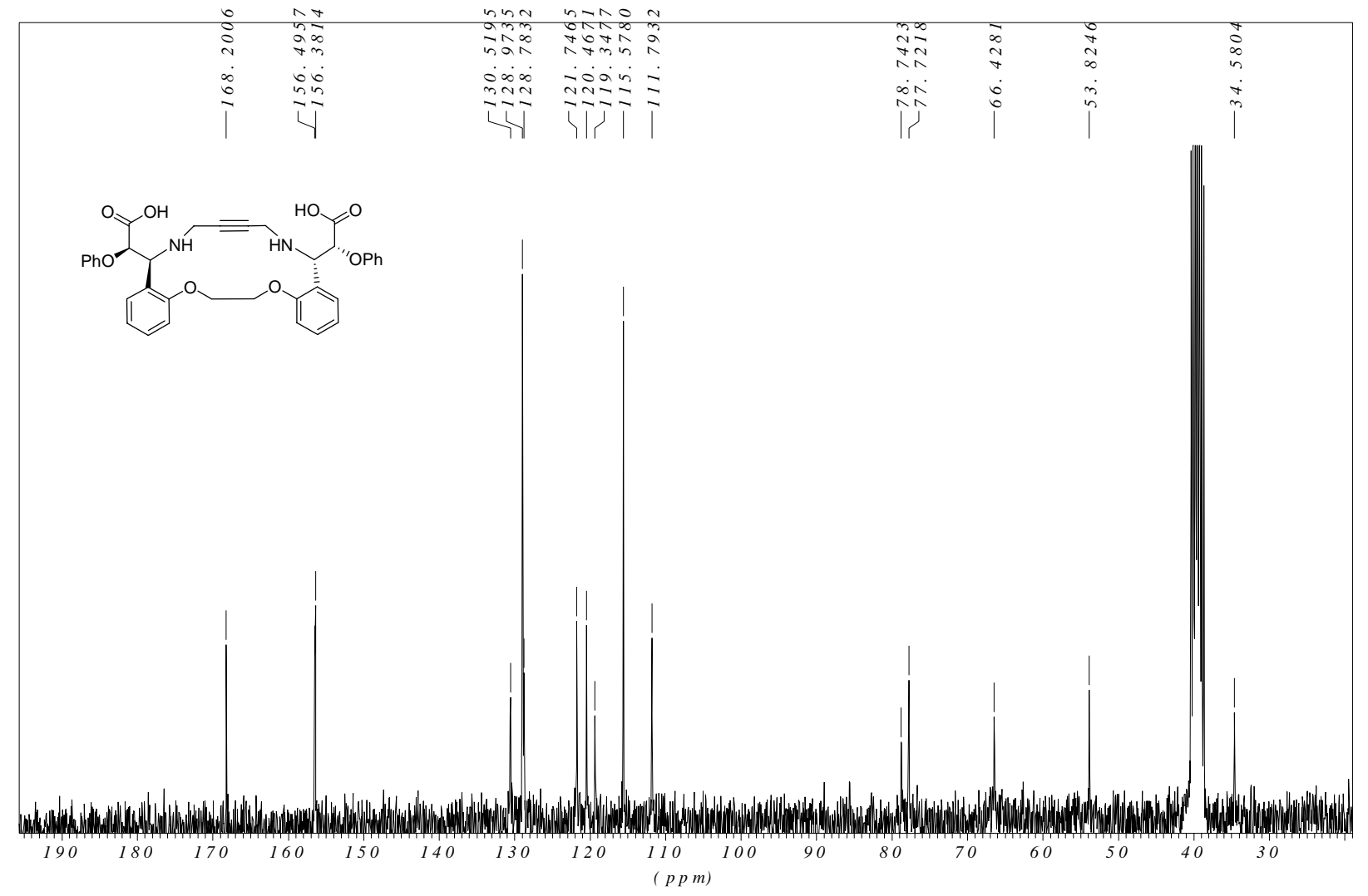


b-Am inoacid 17

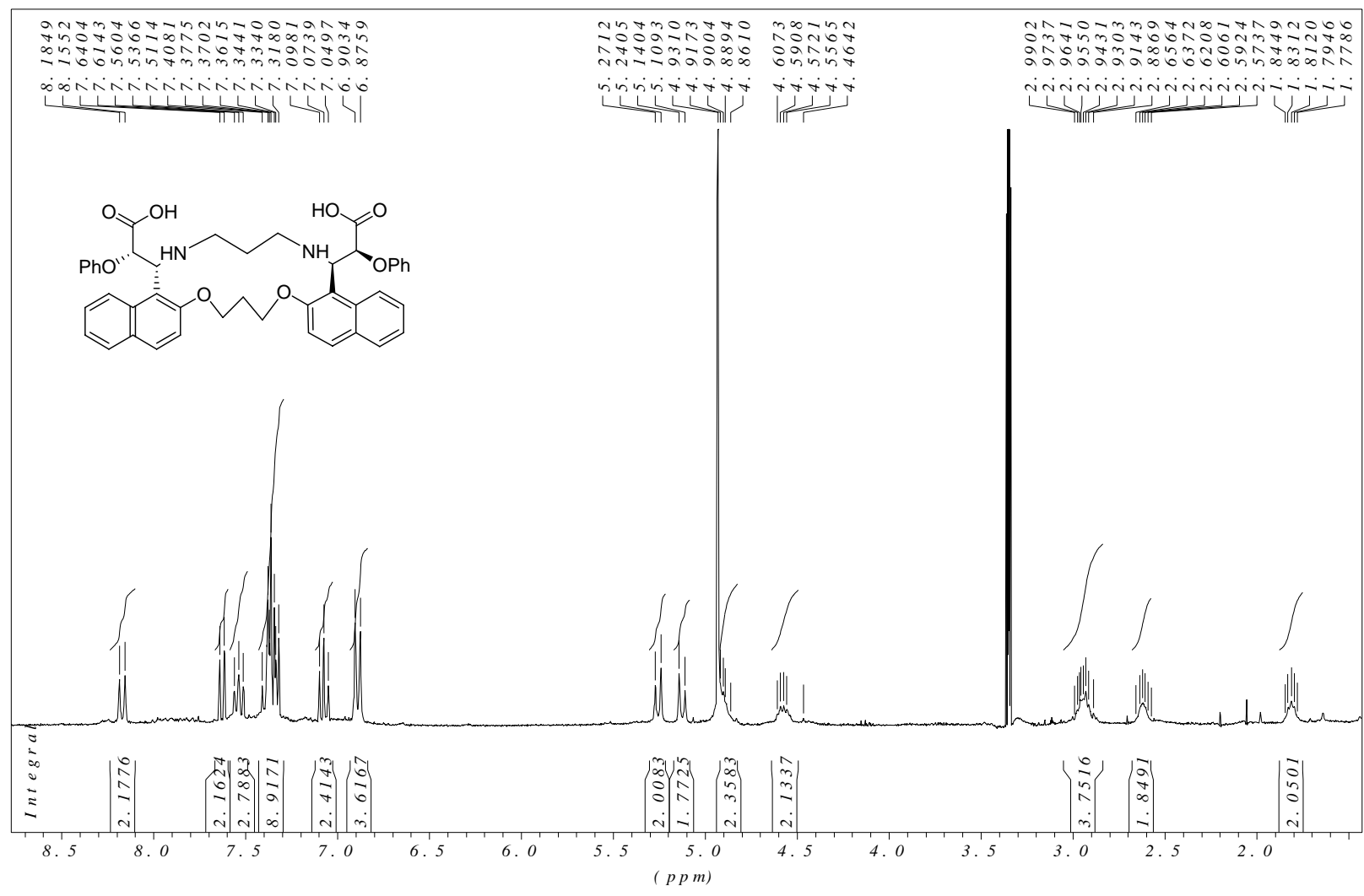

b-A $\mathrm{m}$ in 0 a c id 17
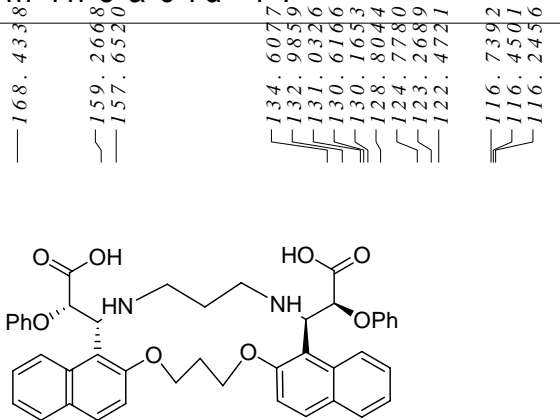

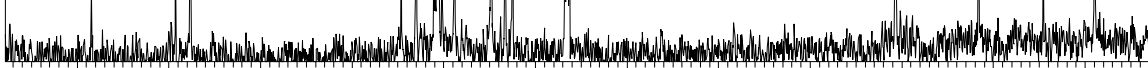

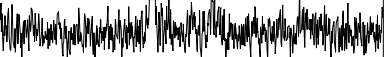

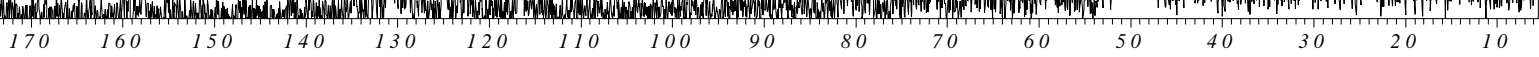




\section{Azetidine 18}
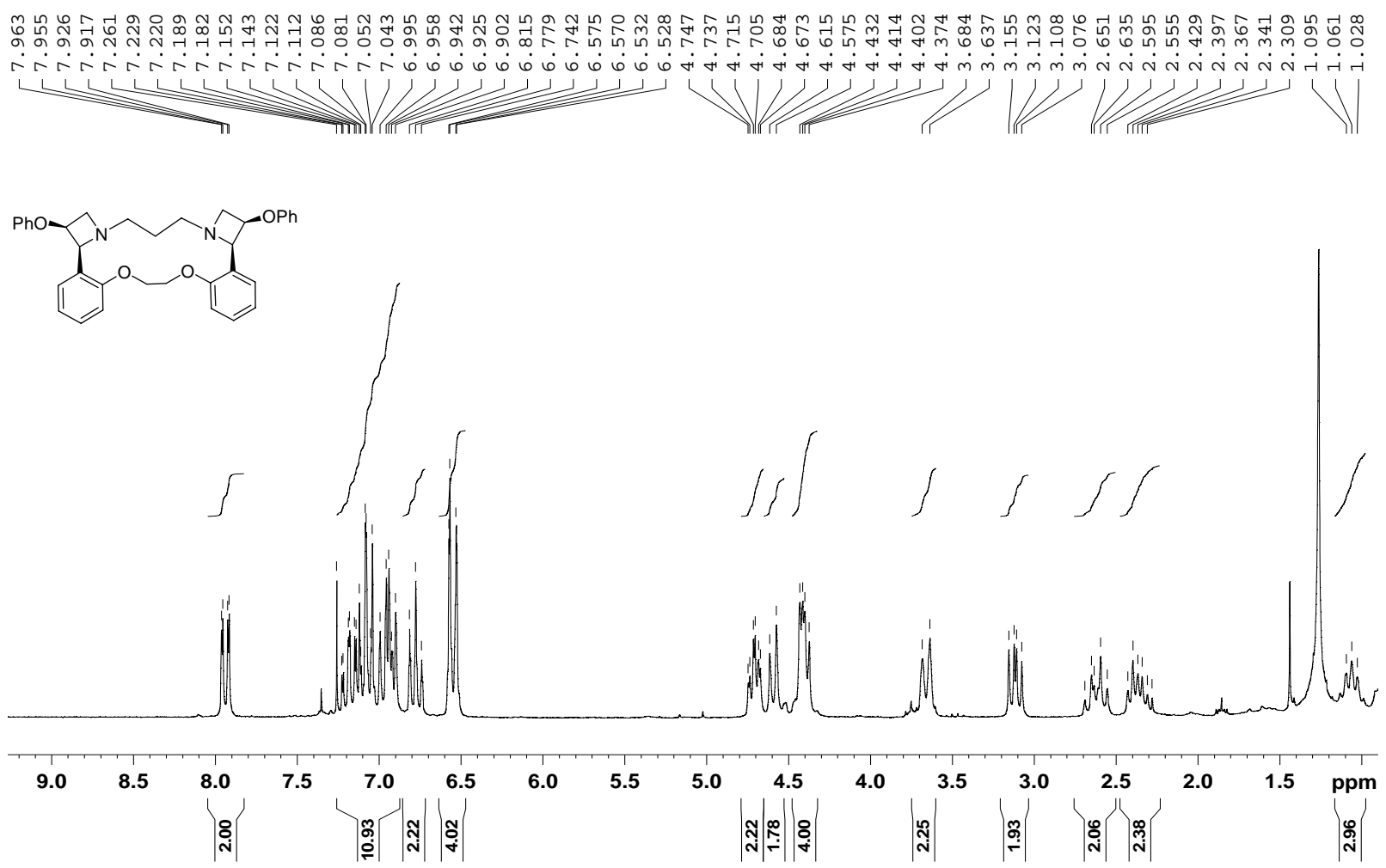

Azetidine 18

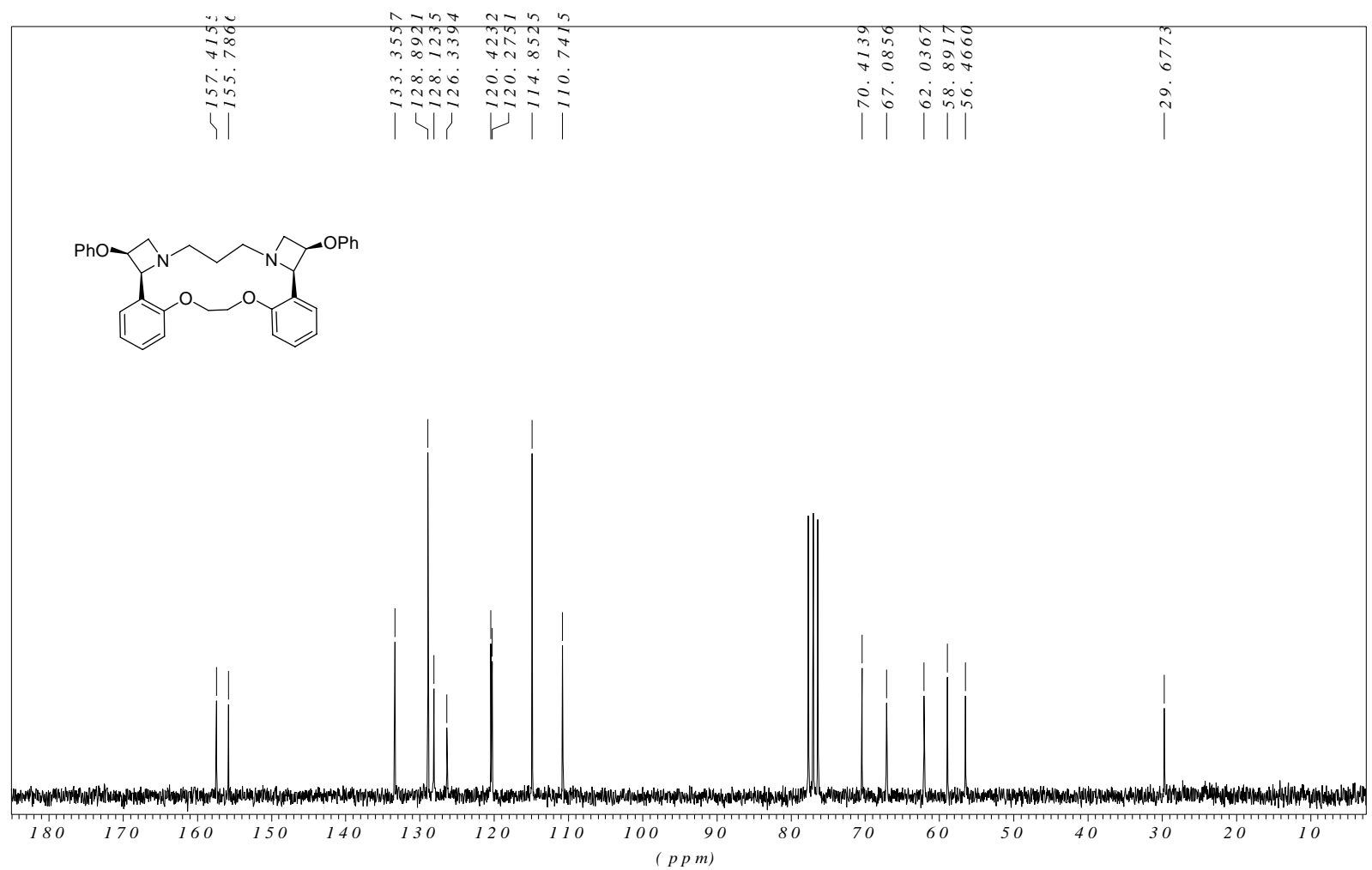


S-22

Azetidine 19

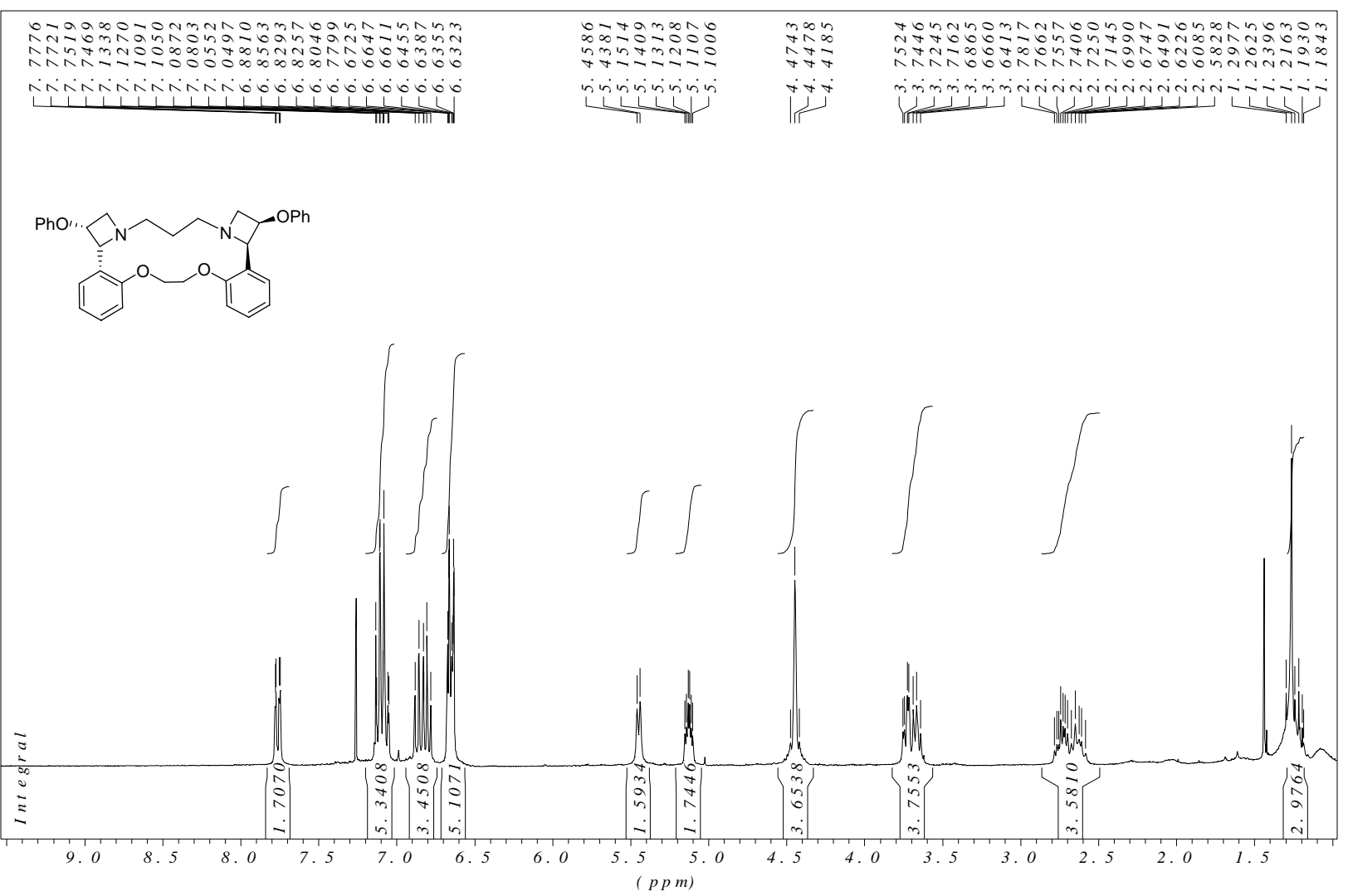

Azetidine 19

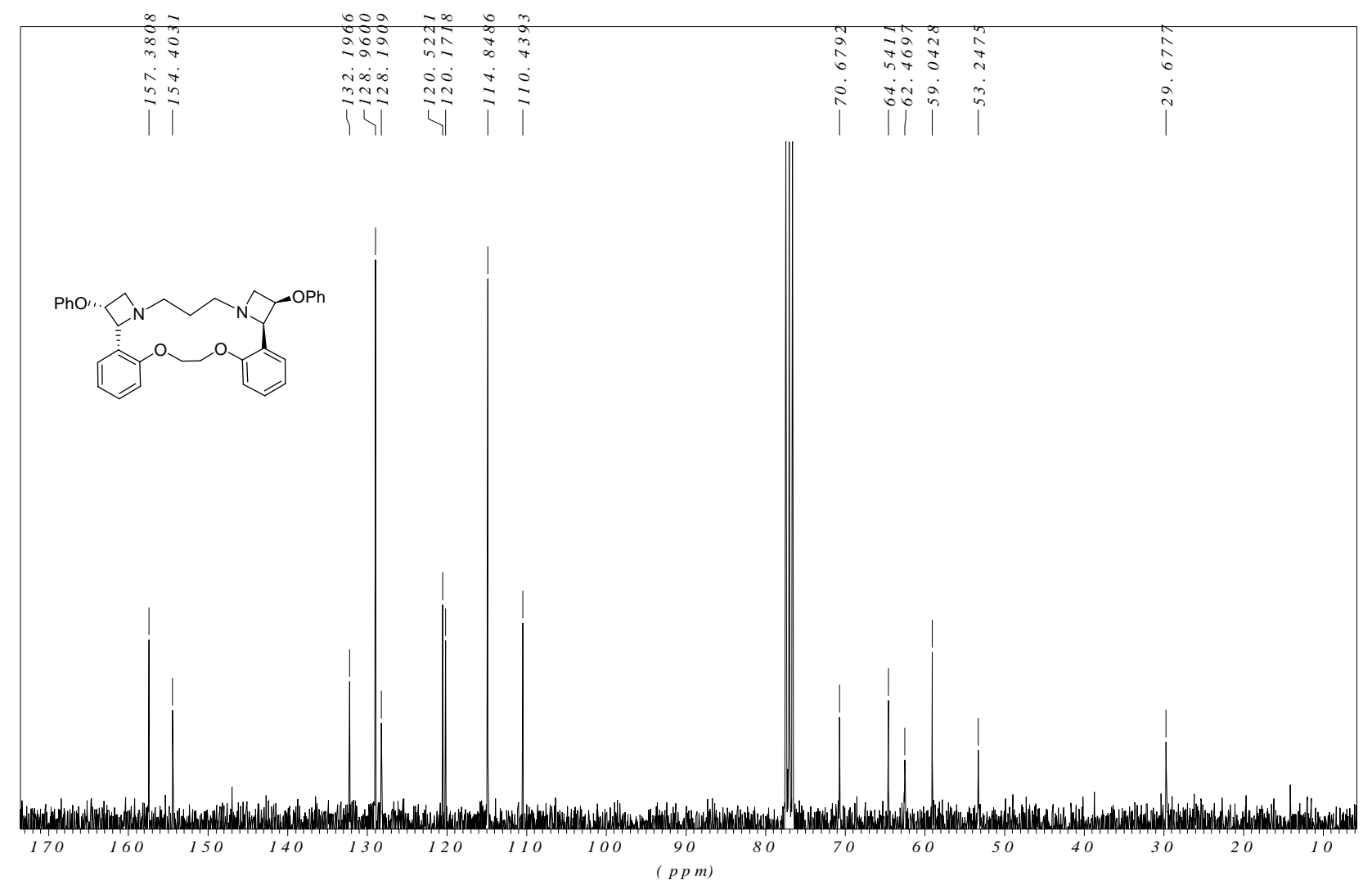


Azetidine 20

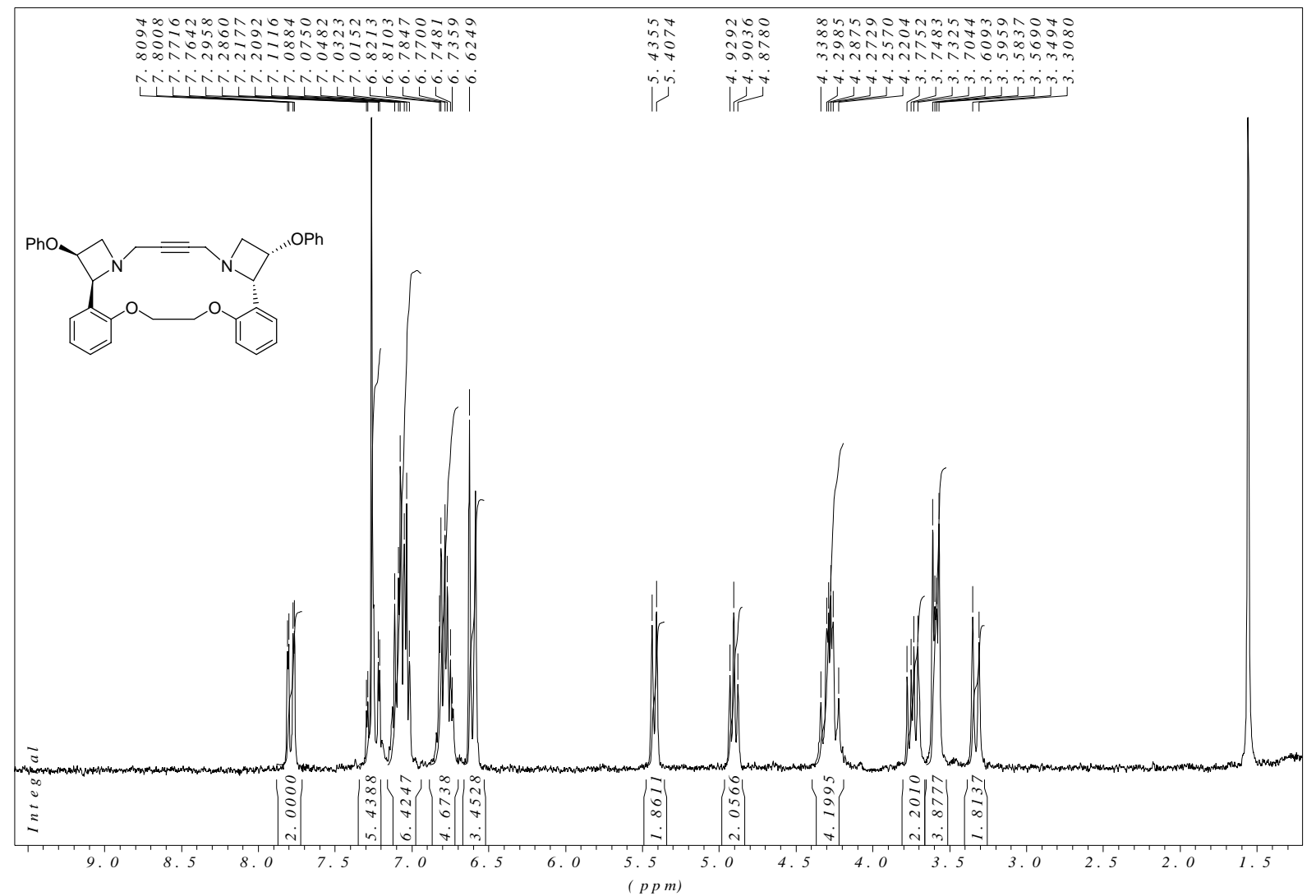

Azetidine 20

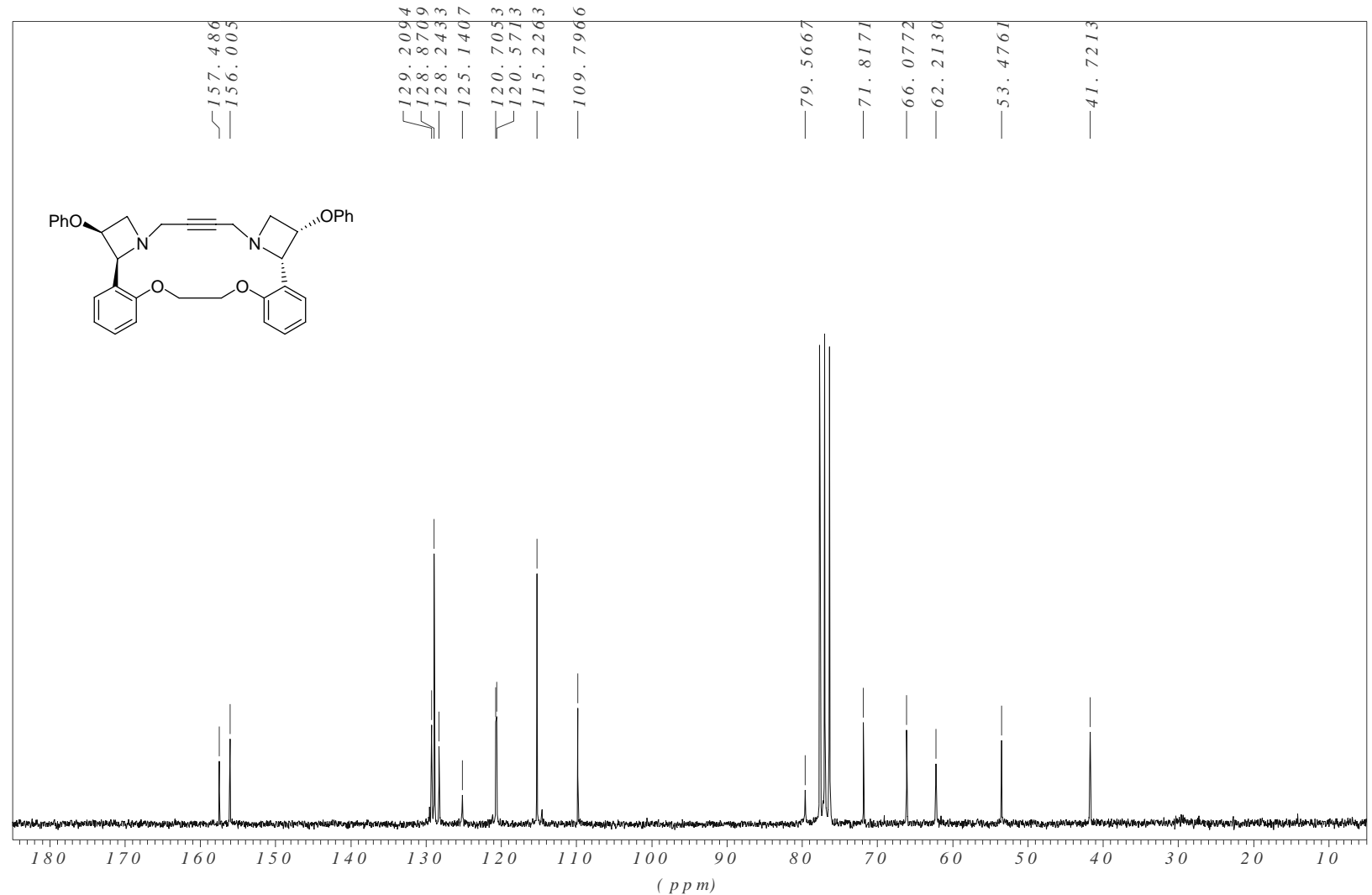


Macrocyclic Amide 21
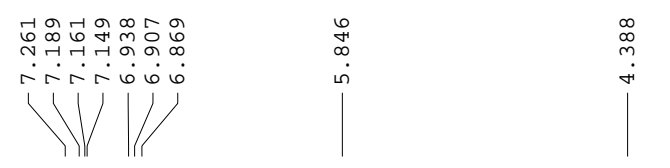

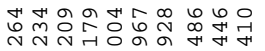

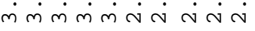

$11 / 1 / 1 / 1$

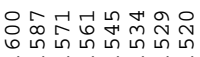

4i

W<smiles></smiles>

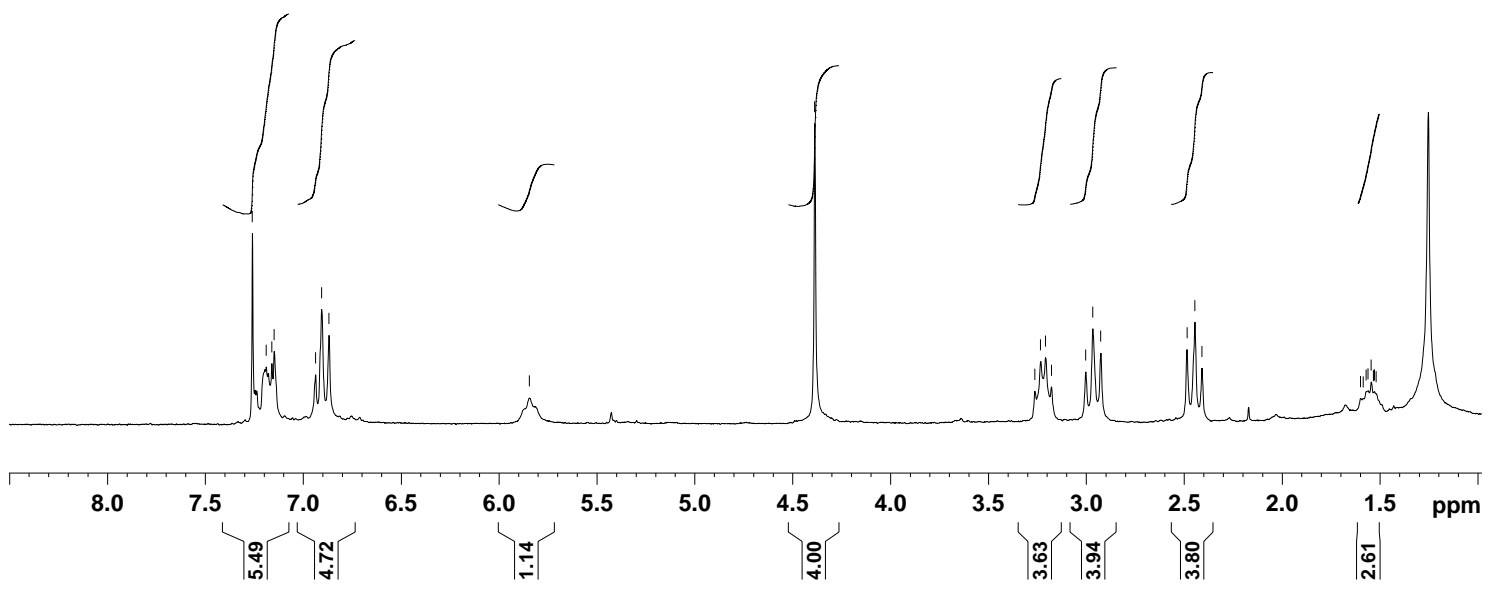

Macrocyclic Amide 21

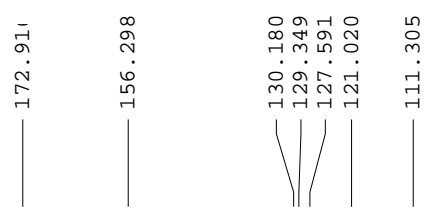

:

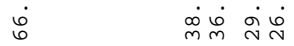

$\mid$

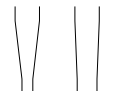<smiles>O=C(CCNCCCNC(=O)Cc1ccccc1COCc1ccccc1)NCCCO</smiles>

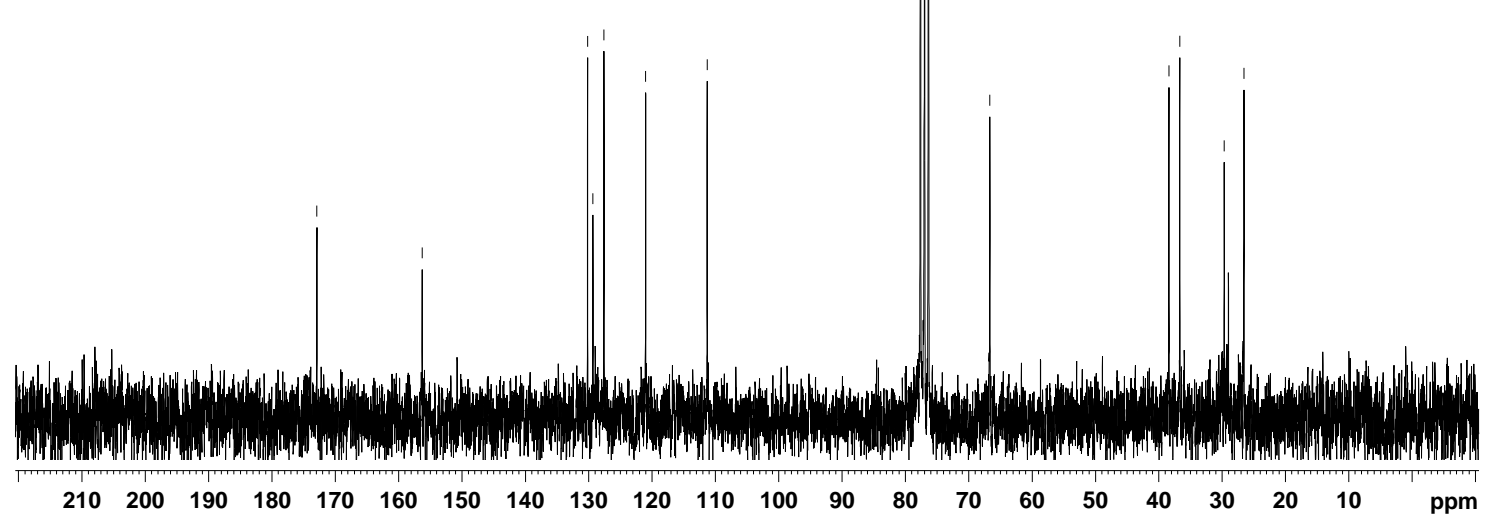


Macrocyclic Amide 22

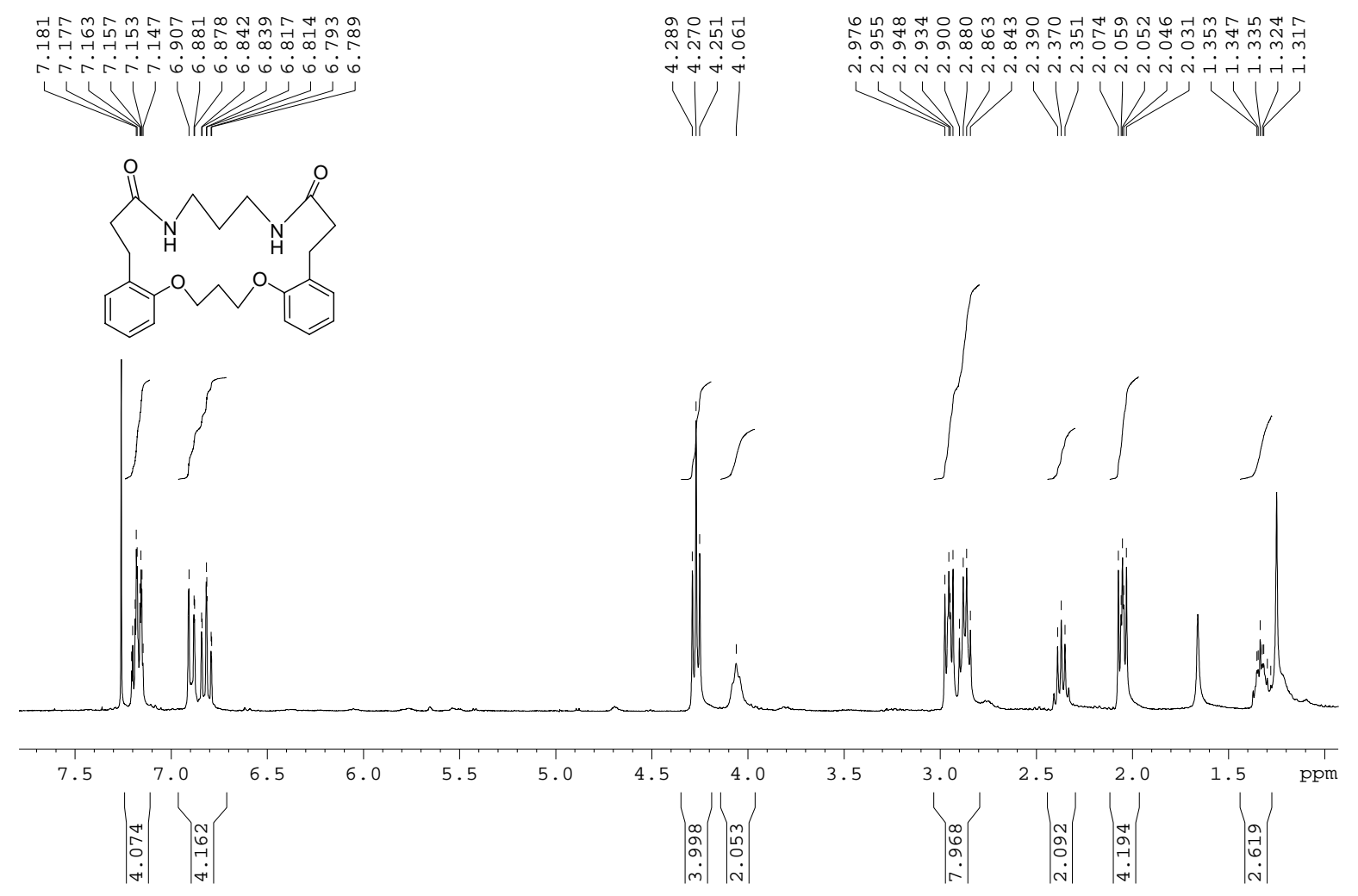

Macrocyclic Amide 22
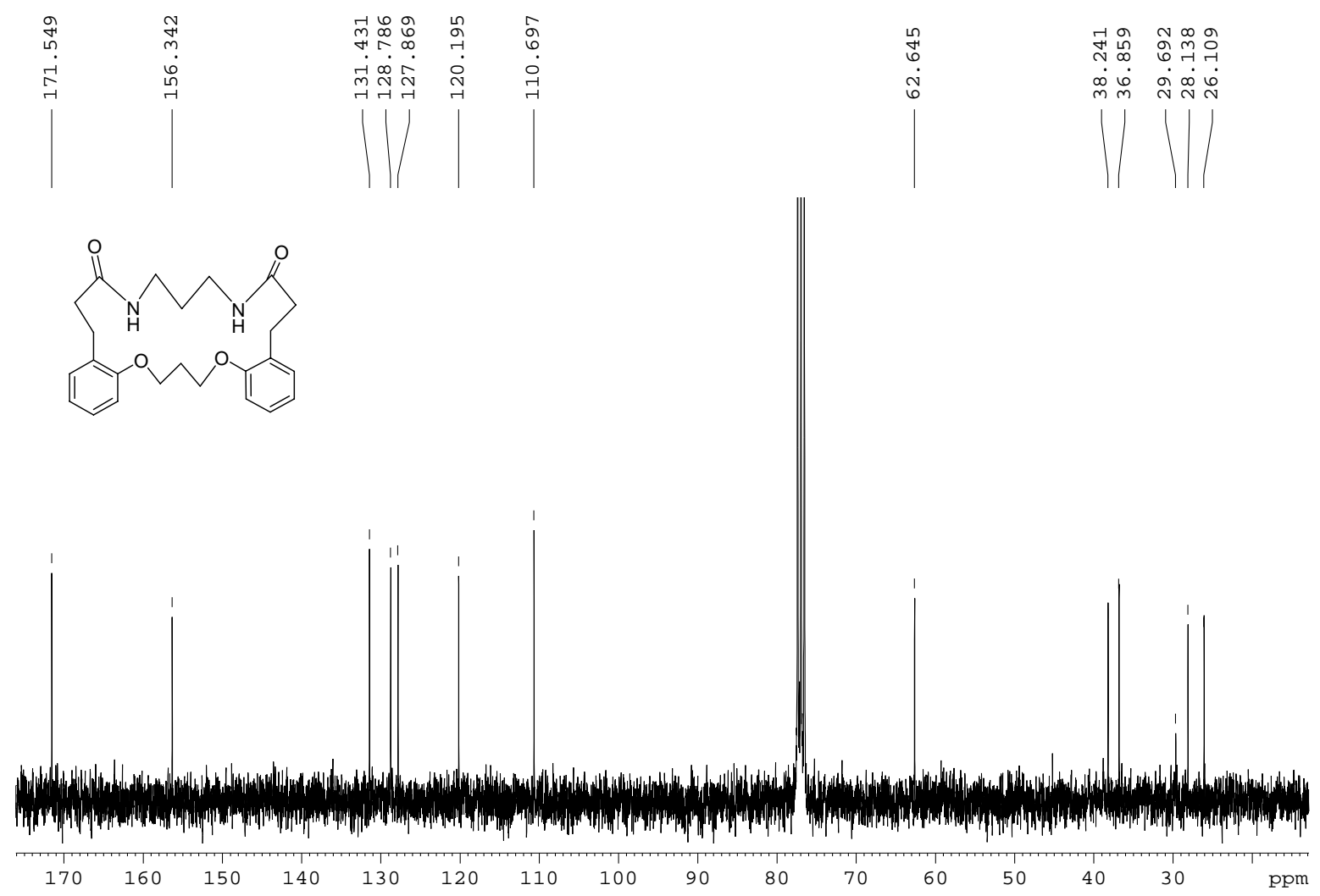